\title{
APPLICATIONS OF THE KUZNETSOV FORMULA ON GL(3) II: THE LEVEL ASPECT
}

\author{
VALENTIN BLOMER, JACK BUTTCANE, AND PÉTER MAGA
}

\begin{abstract}
We develop an explicit Kuznetsov formula on GL(3) for congruence subgroups. Applications include a Lindelöf on average type bound for the sixth moment of GL(3) $L$-functions in the level aspect, an automorphic large sieve inequality, density results for exceptional eigenvalues and density results for Maaß forms violating the Ramanujan conjecture at finite places.
\end{abstract}

\section{INTRODUCTION}

While the toolbox of analytic number theory for classical automorphic forms for congruence subgroups of $\mathrm{SL}_{2}(\mathbb{Z})$ is well developed, much less is known in the case of higher rank groups. It is therefore very desirable to extend the collection of available methods by genuine higher rank tools, such as explicit and for the purpose of analytic number theory user-friendly spectral summation formulae. In this paper we will introduce a version of the powerful Bruggeman-Kuznetsov formula for congruence subgroups of $\mathrm{SL}_{3}(\mathbb{Z})$ and see it in action.

In the situation of the group $\mathrm{SL}_{2}(\mathbb{Z})$ this versatile formula was first developed independently by Bruggeman $[\mathrm{Br}]$ and Kuznetsov [Ku]. Starting with the groundbreaking work of Deshouillers and Iwaniec [DI1], it has become a very attractive tool, among other things because it provides a method for studying averages of Kloosterman sums by automorphic techniques, and has shown itself capable of going sometimes beyond the powerful bounds known for individual Kloosterman sums by the Riemann Hypothesis over finite fields. Classical applications include, among others, density results on exceptional eigenvalues, a proof of Selberg's $3 / 16$ theorem, the best known results on the proportion of critical zeros of the Riemann zeta function, and equidistribution of integral points on spheres.

While such a formula exists in great generality, using the theory of general automorphic forms, a main issue for the purpose of analytic number theory is to make the resulting expression analytically useful. This requires a very good understanding of the integral transforms which relate the test functions on both sides of the formulas at all places which turns out to be a problem both in real and $p$-adic analysis. In this paper the focus is on the level aspect, and a good deal of work is devoted to the investigation of the fine properties of GL(3) Kloosterman sums with prime power moduli, but some of the applications also require more precise information on the archimedean test function than those developed in [Bl].

We proceed to describe the new applications that we group into three sections.

1.1. Moments of $L$-functions. While individual $L$-functions remain rather elusive objects, statistical information in families $\mathcal{F}$ of $L$-functions is often more easily available. The archetypical result in this direction is a statement on the order of magnitude (asymptotic formulae, upper bounds, or sometimes lower bounds) of some moment of a family of $L$-functions

$$
\sum_{f \in \mathcal{F}}|L(1 / 2, f)|^{k}
$$

2000 Mathematics Subject Classification. Primary 11F72, 11F66.

Key words and phrases. Kuznetsov formula, Kloosterman sums, moments of $L$-functions, Lindelöf hypothesis, exceptional eigenvalues, large sieve, Ramanujan conjecture.

The first author was supported by the Volkswagen Foundation and a Starting Grant of the European Research Council. The second author was supported by a Starting Grant of the European Research Council. The third author was supported by a Starting Grant of the European Research Council and OTKA grant no. NK104183. 
at the central point. Trace formulae are particularly suitable to evaluate such moments if the family is given by "spectral properties". Here we consider the GL(3) $L$-functions of large (prime) level $N$ for the congruence subgroup $\Gamma_{0}(N)$, the subgroup of matrices in $\mathrm{SL}_{3}(\mathbb{Z})$ with bottom row congruent to $(0,0, *)$ modulo $N$ acting on the generalized upper half plane $\mathbb{H}_{3}$. This is a subgroup of index $N^{2+o(1)}$ in $\mathrm{SL}_{3}(\mathbb{Z})$. For an $\mathrm{SO}(3)$-invariant subspace of a spherical cuspidal automorphic representation $\pi \subseteq L_{\text {cusp }}^{2}\left(\Gamma_{0}(N) \backslash \mathbb{H}_{3}\right)$ let $\mu_{\pi}$ denote the spectral parameter (Langlands parameter at infinity) of $\pi$, normalized so that it is purely imaginary if the Ramanujan conjecture holds. We fix once and for all a compact set $\Omega \subseteq \mathfrak{a}_{\mathbb{C}}^{*}$, the complexified dual of the Lie algebra of the maximal torus in $\mathrm{PGL}_{3}(\mathbb{R})$. If $\Omega$ is not too small, there are roughly $\asymp \Omega N^{2+o(1)}$ such representations $\pi$ with $\mu_{\pi} \in \Omega$.

It is a fairly straightforward exercise with Kuznetsov formula to prove the following best-possible ("Lindelöf-on-average") bound for the fourth moment:

$$
\sum_{\substack{\pi \subseteq L^{2}\left(\Gamma_{0}(N) \backslash \mathbb{H}_{3}\right) \\ \mu_{\pi} \in \Omega}}|L(1 / 2, \pi)|^{4} \ll N^{2+\varepsilon}
$$

for any $\varepsilon>0$. Here and henceforth in this paper we will apply the usual $\varepsilon$-convention: the letter $\varepsilon$ denotes an arbitrarily small real number, not necessarily the same on each occurrence.

The main work of the paper is devoted to bound a sixth moment in a best-possible fashion. This gives us the possibility to highlight the finer details of the Kloosterman side of the Kuznetsov formula and to give a sample argument how to combine this formula with the rest of the machinery of analytic number theory, such as multiple Poisson summation, estimation of multiple character sums and stationary phase type arguments for the archimedean weight functions.

One of the technical problems is that the Kuznetsov formula - as any spectral summation formula requires a spectrally complete expression. Therefore we have to artificially add the continuous spectrum which unfortunately produces a term of larger of magnitude (the maximal Eisenstein series contribute $N^{5 / 2}$, see Section 5.2). This problem was already faced in the GL(2) situation in [DFI] and [BHM]; in [DFI], a delicate analysis identified a term on the arithmetic side of the Kuznetsov formula that cancelled the continuous spectrum contribution, while in $[\mathrm{BHM}]$ this problem was solved by introducing extra zeros in the Mellin transform of the weight function in the approximate functional equation. Both approaches require extremely subtle and precise information on the archimedean test functions in the Kuznetsov formula that is not easily available in higher rank situations. Here we deal with this problem by twisting the automorphic forms in question with a fixed character in order to kill the unwanted poles incurred by the continuous spectrum. Our first main theorem is as follows.

Theorem 1. Let $N$ be a large prime and let $p$ be a fixed prime. Let $\chi$ be a primitive character modulo $p$ of order $>2$ and let $\Omega \subseteq \mathfrak{a}_{\mathbb{C}}^{*}$. Then

$$
\sum_{\substack{\pi \subseteq L_{\text {cusp }}^{2}\left(\Gamma_{0}(N) \backslash \mathbb{H}_{3}\right) \\ \mu_{\pi} \in \Omega}}|L(1 / 2, \pi \times \chi)|^{6} \ll_{p, \Omega, \varepsilon} N^{2+\varepsilon}
$$

for every $\varepsilon>0$.

1.2. Spectral mean values and a large sieve. Many applications call for an estimate of Fourier coefficients, averaged over the automorphic spectrum. For each $\pi \subseteq L_{\text {cusp }}^{2}\left(\Gamma_{0}(N) \backslash \mathbb{H}_{3}\right)$ we choose a newvector $\varpi$ that we normalize such that its Fourier coefficients, defined in $(2.7)$ and $(2.8)$ below, satisfy $A_{\varpi}(1,1)=1$. The following useful result is the level analogue of [Bl, Theorem 5]:

Theorem 2. Let $n, m, N \in \mathbb{N},(m n, N)=1, \Omega \subseteq \mathfrak{a}_{\mathbb{C}}^{*}$. Then we have

$$
\sum_{\substack{\pi \subseteq L_{\text {cusp }}^{2}\left(\Gamma_{0}(N) \backslash \mathbb{H}_{3}\right) \\ \mu_{\pi} \in \Omega}}\left|A_{\varpi}(n, m)\right|^{2} \ll_{\Omega, \varepsilon}(N m n)^{\varepsilon}\left(N^{2}+N^{1 / 2} n m\right)
$$

for every $\varepsilon>0$. 
The first term on the right hand side is (up to $\varepsilon$ ) the number of terms in the sum which dominates the second term provided $n m \ll N^{3 / 2}$. In particular, in this region the result is best possible and can often be used as a substitute for the Ramanujan conjecture.

A more refined estimate of this type is the following large sieve inequality for the unramified Hecke eigenvalues $\lambda_{\pi}(n)=A_{\varpi}(n, 1),(n, N)=1$.

Theorem 3. Let $N \in \mathbb{N}, \Omega \subseteq \mathfrak{a}_{\mathbb{C}}^{*}, X \geqslant 1$, and let $\alpha(n)$ be a sequence of complex numbers supported on $X \leqslant n \leqslant 2 X$. Then

$$
\sum_{\substack{\pi \subseteq L_{\text {cusp }}^{2}\left(\Gamma_{0}(N) \backslash \mathbb{H}_{3}\right) \\ \mu_{\pi} \in \Omega}}\left|\sum_{\substack{X \leqslant n \leqslant 2 X \\(n, N)=1}} \lambda_{\pi}(n) \alpha(n)\right|^{2} \ll \Omega, \varepsilon(N X)^{\varepsilon}\left(N^{2}+X^{2} N^{1 / 2}\right)\|\alpha\|^{2}
$$

for every $\varepsilon>0$.

This is in the spirit of the celebrated large sieve inequalities of [DI1]. It should be compared with the case $n=3$ of [Ve] which requires $X \leqslant N^{1 / 4}$ to be optimal whereas our result covers the much larger range $X \leqslant N^{3 / 4}$ (cf. also [DK] for a different large sieve inequality). This shows the advantage of using a powerful tool like the Kuznetsov formula as opposed to the soft methods in [Ve] which on the other hand generalize directly to $\mathrm{GL}(n)$.

1.3. Exceptional eigenvalues and the Ramanujan conjecture. The Ramanujan conjecture is one of the central open problems in the theory of automorphic forms, known only for cohomological forms. In analytic number theory it is often important to control the degree to which the Ramanujan conjecture is violated, and to show that this cannot happen too frequently. The following theorems provide bounds for the density of forms violating the Ramanujan conjecture at a given place, and we will show in particular that in a quantitative sense almost all Maaß forms satisfy the Ramanujan conjecture at a given place. In the eigenvalue aspect this has been investigated in $[\mathrm{BBR}]$. We start with the archimedean place and show the following density result for exceptional Maaß forms of large level:

Theorem 4. Let $N$ be a prime and $\Omega \subseteq \mathfrak{a}_{\mathbb{C}}^{*}$. Then

$$
\sum_{\substack{\pi \subseteq L_{\text {cusp }}^{2}\left(\Gamma_{0}(N) \backslash \mathbb{H}_{3}\right) \\ \mu_{\pi} \in \Omega}} N^{4\left\|\Re \mu_{\pi}\right\|} \ll_{\Omega, \varepsilon} N^{2+\varepsilon}
$$

for every $\varepsilon>0$.

Here and in the following, $\|$.$\| denotes the maximum norm. The Jacquet-Shalika [JS] bounds imply$ $\left\|\Re \mu_{\pi}\right\| \leqslant 1 / 2$ while the Kim-Sarnak method shows $\left\|\Re \mu_{\pi}\right\| \leqslant 5 / 14$. Our result recovers (essentially) the Jacquet-Shalika bounds, but it shows much more: exceptional Maaß forms occur less and less frequent, the more the Ramanujan conjecture at infinity is violated.

A similar result can be obtained for a fixed finite place. Let $\alpha_{\pi}(p)$ denote the Satake parameter of a representation $\pi$ at $p$. The Ramanujan conjecture states that all three entries of $\alpha_{\pi}(p)$ have absolute value one.

Theorem 5. Let $N \in \mathbb{N}$, fix a prime $p \nmid N$ and let $\delta>0$. Let $\Omega \subseteq \mathfrak{a}_{\mathbb{C}}^{*}$. Then there exists $\eta>0$ (depending on $\delta$ and $p$ ) such that

$$
\#\left\{\pi \subseteq L_{\text {cusp }}^{2}\left(\Gamma_{0}(N) \backslash \mathbb{H}_{3}\right): \mu_{\pi} \in \Omega,\left\|\alpha_{\pi}(p)\right\| \geqslant 1+\delta\right\} \ll_{\Omega, \delta, p}\left(N^{2}\right)^{1-\eta} .
$$

\section{The Kuznetsov formula for Congruence Subgroups of $\mathrm{SL}_{3}(\mathbb{Z})$}

In this section we state and prove the Kuznetsov formula and correct a small error in the statement of the formula in $[\mathrm{Bl}]$. This requires a bit of notational preparation. Let $N \in \mathbb{N}$ be the level. We follow the 
approach in [Bl] and compute the inner product of two Poincaré series in two ways. Let $F:(0, \infty)^{2} \rightarrow \mathbb{C}$ be a smooth compactly supported function. Let

$$
F^{*}\left(y_{1}, y_{2}\right):=F\left(y_{2}, y_{1}\right)
$$

For two positive integers $m_{1}, m_{2}$ and $z=\left(\begin{array}{ccc}1 & x_{2} & x_{3} \\ & 1 & x_{1} \\ & & 1\end{array}\right)\left(\begin{array}{ccc}y_{1} y_{2} & \\ & y_{1} & \\ & & 1\end{array}\right) \in \mathbb{H}_{3}$ let

$$
\mathcal{F}_{m_{1}, m_{2}}(z):=e\left(m_{1} x_{1}+m_{2} x_{2}\right) F\left(m_{1} y_{1}, m_{2} y_{2}\right) .
$$

Then we consider the following Poincaré series:

$$
P_{m_{1}, m_{2}}(z):=\sum_{\gamma \in \Gamma_{\infty} \backslash \Gamma_{0}(N)} \mathcal{F}_{m_{1}, m_{2}}(\gamma z)
$$

where $\Gamma_{\infty}$ is the subgroup of unipotent upper triangular matrices. The Fourier expansion of these functions features Kloosterman sums and their archimedean analogues, certain special functions given by an integral representation. The three non-trivial terms in the Kuznetsov formula are attached to the elements $w_{4}=$ $\left(\begin{array}{ll}{ }_{1} & 1\end{array}\right), w_{5}=\left(\begin{array}{ll}1 & 1 \\ & 1\end{array}\right)$ and $w_{6}=\left({ }_{1}^{-1}{ }^{1}\right)$ in the Weyl group. Correspondingly, for $m_{1}, m_{2}, n_{1}, n_{2} \in \mathbb{Z} \backslash\{0\}$ we define

$$
\tilde{S}\left(m_{1}, n_{1}, n_{2} ; D_{1}, D_{2}\right):=\sum_{\substack{C_{1}\left(\bmod D_{1}\right), C_{2}\left(\bmod D_{2}\right) \\\left(C_{1}, D_{1}\right)=\left(C_{2}, D_{2} / D_{1}\right)=1}} e\left(n_{1} \frac{\bar{C}_{1} C_{2}}{D_{1}}+n_{2} \frac{\bar{C}_{2}}{D_{2} / D_{1}}+m_{1} \frac{C_{1}}{D_{1}}\right),
$$

for $D_{1} \mid D_{2}$ and

$$
\begin{aligned}
& S^{(N)}\left(m_{1}, m_{2}, n_{1}, n_{2} ; D_{1}, D_{2}\right) \\
& =\sum_{B_{1}, C_{1}\left(\bmod D_{1}\right)} e\left(\frac{m_{1} B_{1}+n_{1}\left(Y_{1} D_{2}-Z_{1} B_{2}\right)}{D_{1}}+\frac{m_{2} B_{2}+n_{2}\left(Y_{2} D_{1}-Z_{2} B_{1}\right)}{D_{2}}\right) \\
& \begin{array}{c}
B_{2}, C_{2}\left(\bmod D_{2}\right) \\
D_{1} C_{2}+B_{1} B_{2}+D_{2} C_{1} \equiv 0\left(\bmod D_{1} D_{2}\right)
\end{array} \\
& \left(B_{j}, C_{j}, D_{j}\right)=1, N \mid B_{1}
\end{aligned}
$$

for $N\left|D_{1}, N\right| D_{2}$, where $Y_{j} B_{j}+Z_{j} C_{j} \equiv 1\left(\bmod D_{j}\right)$ for $j=1,2$. The latter is almost the same sum as in [BFG, Section 4] for level 1 except for the additional divisibility condition $N \mid B_{1}$. Note, however, that $N \mid B_{1} B_{2}$ is automatic (since $N \mid D_{1}, D_{2}$ ), so the additional condition $N \mid B_{1}$ is relatively minor.

The archimedean functions don't see the additional level and are identical to the level 1 case. For $\epsilon \in\{ \pm 1\}$ or $\{ \pm 1\}^{2}, F$ as above and $A_{1}, A_{2}>0$ we define

$$
\begin{aligned}
& \tilde{\mathcal{J}}_{\epsilon ; F}(A)=A^{-2} \int_{0}^{\infty} \int_{0}^{\infty} \int_{-\infty}^{\infty} \int_{-\infty}^{\infty} e\left(-\epsilon A x_{1} y_{1}\right) e\left(y_{2} \cdot \frac{x_{1} x_{2}}{x_{1}^{2}+1}\right) e\left(\frac{A}{y_{1} y_{2}} \cdot \frac{x_{2}}{x_{1}^{2}+x_{2}^{2}+1}\right) \\
& \times F\left(y_{2} \cdot \frac{\sqrt{x_{1}^{2}+x_{2}^{2}+1}}{x_{1}^{2}+1}, \frac{A}{y_{1} y_{2}} \cdot \frac{\sqrt{x_{1}^{2}+1}}{x_{1}^{2}+x_{2}^{2}+1}\right) \overline{F\left(A y_{1}, y_{2}\right)} d x_{1} d x_{2} \frac{d y_{1} d y_{2}}{y_{1} y_{2}^{2}} \\
& \mathcal{J}_{\epsilon ; F}\left(A_{1}, A_{2}\right)=\left(A_{1} A_{2}\right)^{-2} \int_{0}^{\infty} \int_{0}^{\infty} \int_{-\infty}^{\infty} \int_{-\infty}^{\infty} \int_{-\infty}^{\infty} e\left(-\epsilon_{1} A_{1} x_{1} y_{1}-\epsilon_{2} A_{2} x_{2} y_{2}\right) \\
&\left.\times e\left(-\frac{A_{2}}{y_{2}} \cdot \frac{x_{1} x_{3}+x_{2}}{x_{3}^{2}+x_{2}^{2}+1}\right) e\left(-\frac{A_{1}}{y_{1}} \cdot \frac{x_{2}\left(x_{1} x_{2}-x_{3}\right)+x_{1}}{\left(x_{1} x_{2}-x_{3}\right)^{2}+x_{1}^{2}+1}\right) \frac{\sqrt{x_{3}^{2}+x_{2}^{2}+1}}{F\left(A_{1} y_{1}, A_{2} y_{2}\right)}\right) d x_{1} d x_{2} d x_{3} \frac{d y_{1} d y_{2}}{y_{1} y_{2}} . \\
& \quad \times F\left(\frac{A_{2}}{y_{2}} \cdot \frac{\sqrt{\left(x_{1} x_{2}-x_{3}\right)^{2}+x_{1}^{2}+1}}{x_{3}^{2}+x_{2}^{2}+1}, \frac{A_{1}}{y_{1}} \cdot \frac{\sqrt{\left.x_{1} x_{2}-x_{3}\right)^{2}+x_{1}^{2}+1}}{\left(x_{1}\right.}\right)
\end{aligned}
$$


Next we define for $\mu \in \mathfrak{a}_{\mathbb{C}}^{*}$ and $y_{1}, y_{2}>0$ the (slightly renormalized) Whittaker function as in [Bl, (2.15)] by its double Mellin transform

$$
\begin{aligned}
\tilde{W}_{\mu}\left(y_{1}, y_{2}\right) & =\frac{y_{1} y_{2} \pi^{\frac{3}{2}}}{\left|\Gamma\left(\frac{1}{2}\left(1+i \Im\left(\mu_{1}+2 \mu_{2}\right)\right)\right) \Gamma\left(\frac{1}{2}\left(1+i \Im\left(\mu_{1}-\mu_{2}\right)\right)\right) \Gamma\left(\frac{1}{2}\left(1+i \Im\left(2 \mu_{1}+\mu_{2}\right)\right)\right)\right|} \\
& \times \frac{1}{(2 \pi i)^{2}} \int_{(1)} \int_{(1)} \frac{\prod_{j=1}^{3} \Gamma\left(\frac{1}{2}\left(s_{1}+\mu_{j}\right)\right) \prod_{j=1}^{3} \Gamma\left(\frac{1}{2}\left(s_{2}-\mu_{j}\right)\right)}{4 \pi^{s_{1}+s_{2}} \Gamma\left(\frac{1}{2}\left(s_{1}+s_{2}\right)\right)} y_{1}^{-s_{1}} y_{2}^{-s_{2}} d s_{1} d s_{2} .
\end{aligned}
$$

For a (not necessarily cuspidal) automorphic form $\varpi$ of level $N$ and spectral parameter $\mu$ we define the Fourier coefficient $\tilde{A}_{\varpi}\left(m_{1}, m_{2}\right)\left(m_{1}, m_{2} \neq 0\right)$ by

$$
\int_{0}^{1} \int_{0}^{1} \int_{0}^{1} \varpi(z) e\left(-m_{1} x_{1}-m_{2} x_{2}\right) d x_{1} d x_{2} d x_{3}=\frac{\tilde{A}_{\varpi}\left(m_{1}, m_{2}\right)}{\left|m_{1} m_{2}\right|} \tilde{W}_{\mu}\left(\left|m_{1}\right| y_{1},\left|m_{2}\right| y_{2}\right) .
$$

To ease notation, we will denote by $\{\varpi\}$ an orthonormal basis of automorphic forms of level $N$, cuspidal or Eisenstein series, containing all cuspidal newvectors, and we denote by $\int_{(N)} d \varpi$ a combined sum/integral over the complete spectrum of level $N$. The relevant spectral decomposition is a special case of Langlands' general theory, see e.g. [Ar] for a convenient summary in adelic language. By Hecke theory, we can and will assume that all $\varpi$ are eigenfunctions of the Hecke algebra coprime to $N$. Since

$$
\Gamma_{0}(N) \operatorname{diag}\left(m_{0} m_{1} m_{2}, m_{0} m_{1}, m_{0}\right) \Gamma_{0}(N)=\Gamma_{0}(1) \operatorname{diag}\left(m_{0} m_{1} m_{2}, m_{0} m_{1}, m_{0}\right) \Gamma_{0}(1)
$$

for $\left(m_{0} m_{1} m_{2}, N\right)=1$, this is just the unramified Hecke algebra that satisfies the usual GL(3) Hecke relations as in [Go, Theorem 6.4.11]. The proof of [Go, Theorem 6.4.11] also shows that if $\tilde{A}_{\varpi}(1,1)=0$, then $\tilde{A}_{\varpi}\left(m_{1}, m_{2}\right)=0$ whenever $\left(m_{1} m_{2}, N\right)=1$. If $\tilde{A}_{\varpi}(1,1) \neq 0$, which is the case in particular for newvectors $\varpi$, we write

$$
A_{\varpi}\left(m_{1}, m_{2}\right)=\tilde{A}_{\varpi}\left(m_{1}, m_{2}\right) / \tilde{A}_{\varpi}(1,1),
$$

in which case the normalized Fourier coefficients $A_{\varpi}\left(m_{1}, m_{2}\right)$ satisfy the multiplicativity relations of [Go, Theorem 6.4.11]. If $\tilde{A}_{\varpi}(1,1)=0$, we simply write $A_{\varpi}\left(m_{1}, m_{2}\right)=\tilde{A}_{\varpi}\left(m_{1}, m_{2}\right)$ and remark already at this place that for such $\varpi$ only vanishing Fourier coefficients will come up in our analysis (which, in a trivial way, satisfy the Hecke relations), so that the normalization is irrelevant. For notational consistency we write $N(\varpi)=\tilde{A}_{\varpi}(1,1)^{2}$ if $\tilde{A}_{\varpi}(1,1) \neq 0$ and $N(\varpi)=1$ otherwise.

Rankin-Selberg theory shows (see e.g. [Bl, Lemma 1]) that for a cuspidal newform $\varpi \in \pi$ one has

$$
\mathcal{N}(\varpi) \asymp\left[\mathrm{SL}_{3}(\mathbb{Z}): \Gamma_{0}(N)\right] \cdot \underset{s=1}{\operatorname{res}} \sum_{m_{1}, m_{2}} \frac{\left|A_{\varpi}\left(m_{1}, m_{2}\right)\right|^{2}}{m_{1}^{2 s} m_{2}^{s}}
$$

and it follows from [Li, Theorem 2] that

$$
\mathcal{N}(\varpi) \ll N^{2}\left(N\left(1+\left|\mu_{\pi}\right|\right)\right)^{\varepsilon} .
$$

We define an inner product on $(0, \infty)^{2}$ by

$$
\langle f, g\rangle:=\int_{0}^{\infty} \int_{0}^{\infty} f\left(y_{1}, y_{2}\right) \overline{g\left(y_{1}, y_{2}\right)} \frac{d y_{1} d y_{2}}{\left(y_{1} y_{2}\right)^{3}} .
$$

With this notation we are ready to state our version of the Kuznetsov formula.

Theorem 6. Let $F$ be a compactly supported test function with $F^{*}$ as in (2.1). Let $N, n_{1}, n_{2}, m_{1}, m_{2} \in \mathbb{N}$. Then

$$
\int_{(N)} \frac{\overline{A_{\varpi}\left(n_{1}, n_{2}\right)} A_{\varpi}\left(m_{1}, m_{2}\right)}{\mathcal{N}(\varpi)}\left|\left\langle\tilde{W}_{\mu_{\pi}}, F\right\rangle\right|^{2} d \varpi=\Delta+\Sigma_{4}+\Sigma_{5}+\Sigma_{6}
$$


where

$$
\begin{aligned}
\Delta & =\delta_{n_{1}, m_{1}} \delta_{n_{2}, m_{2}}\|F\|^{2}, \\
\Sigma_{4} & =\sum_{\epsilon= \pm 1} \sum_{\substack{N D_{2} \mid D_{1} \\
n_{2} D_{1}=m_{1} D_{2}^{2}}} \frac{\tilde{S}\left(\epsilon m_{2}, n_{2}, n_{1}, D_{2}, D_{1}\right)}{D_{1} D_{2}} \tilde{\mathcal{J}}_{\epsilon ; F^{*}}\left(\sqrt{\frac{n_{1} n_{2} m_{2}}{D_{1} D_{2}}}\right), \\
\Sigma_{5} & =\sum_{\epsilon= \pm 1} \sum_{\substack{N\left|D_{1}\right| D_{2} \\
n_{1} D_{2}=m_{2} D_{1}^{2}}} \frac{\tilde{S}\left(\epsilon m_{1}, n_{1}, n_{2}, D_{1}, D_{2}\right)}{D_{1} D_{2}} \tilde{\mathcal{J}}_{\epsilon ; F}\left(\sqrt{\frac{n_{1} n_{2} m_{1}}{D_{1} D_{2}}}\right), \\
\Sigma_{6} & =\sum_{\epsilon=\epsilon_{2}= \pm 1} \sum_{N\left|D_{1}, N\right| D_{2}} \frac{S^{(N)}\left(\epsilon_{2} m_{2}, \epsilon_{1} m_{1}, n_{1}, n_{2}, D_{1}, D_{2}\right)}{D_{1} D_{2}} \mathcal{J}_{\epsilon ; F}\left(\frac{\sqrt{n_{2} m_{1} D_{1}}}{D_{2}}, \frac{\sqrt{n_{1} m_{2} D_{2}}}{D_{1}}\right) .
\end{aligned}
$$

Remarks: (1) In [Bl], the first two entries in the long Weyl element Kloosterman sum are mistakenly interchanged, cf. [BFG, p. 64].

(2) The Fourier coefficients of Eisenstein series for $\Gamma_{0}(N) \subseteq \mathrm{SL}_{3}(\mathbb{Z})$ for all indices are computed in detail in $[\mathrm{Ba}]$.

(3) Note that there is a small asymmetry in the definition of $\Sigma_{4}$ and $\Sigma_{5}$. If $\left(n_{1} m_{1}, N\right)=1$, then the summation condition in $\Sigma_{4}$ is equivalent to $D_{1}=N^{2} d_{1} d_{2}, D_{2}=N d_{2}, n_{2} d_{1}=m_{1} d_{2}$, while the summation condition in $\Sigma_{5}$ is equivalent to $D_{1}=N d_{1}, D_{2}=N^{2} d_{1} d_{2}, n_{1} d_{2}=m_{2} d_{1}$, so complete symmetry between $\Sigma_{4}$ and $\Sigma_{5}$ is restored.

Proof. This is exactly as in [Bl, Proposition 4] by computing the inner product of two level $N$ Poincaré series by unfolding and by spectral decomposition and then comparing both expressions. We only have to verify that the definition of our Kloosterman sums agrees with the Fourier expansion of the level $N$ Poincaré series. The exponential sums appearing in the latter are most easily, but abstractly, defined in terms of the Bruhat decomposition, so the procedure is to enumerate the terms in the sum using the Plücker coordinates, determine the summand as a function of the Plücker coordinates by writing out the Bruhat decomposition of each term, and then verify that the summand only depends on the residue classes of the Plücker coordinates.

Let $U(R)$ be the group of upper-triangular matrices with ones on the diagonal and entries in the ring $R, W$ the Weyl group and $V$ the diagonal orthogonal matrices of $\mathrm{SL}_{3}(\mathbb{Z})$. We also need the decomposition of $U(R)$ by $w \in W$, so set

$$
U_{w}(R)=\left(w^{-1} U(R) w\right) \cap U(R), \quad \bar{U}_{w}(R)=\left(w^{-1} U(R)^{t} w\right) \cap U(R) .
$$

Define characters of $U(\mathbb{R})$ by

$$
\psi_{n_{1}, n_{2}}\left(\begin{array}{ccc}
1 & x_{2} & * \\
& 1 & x_{1} \\
& & 1
\end{array}\right)=e\left(n_{1} x_{1}+n_{2} x_{2}\right)
$$

where we assume $n_{1}, n_{2} \in \mathbb{Z}$. Then the Bruhat decomposition of some $\gamma \in \mathrm{SL}_{3}(\mathbb{Z})$ takes the form $\gamma=$ $b c v w b^{\prime}$ with $w \in W, v \in V, b, b^{\prime} \in U(\mathbb{R})$ and $c=\operatorname{diag}\left(1 / c_{2}, c_{2} / c_{1}, c_{1}\right)$ for some $c_{1}, c_{2} \in \mathbb{N}$. The Bruhat decomposition is only defined up to an element of $U_{w}(\mathbb{R})$.

Now let $w \in W, n_{1}, n_{2}, m_{1}, m_{2} \in \mathbb{Z}$ and $c=\operatorname{diag}\left(1 / c_{2}, c_{2} / c_{1}, c_{1}\right)$ as before. If the compatibility condition

$$
\psi_{n_{1}, n_{2}}\left((c w) u(c w)^{-1}\right) \psi_{m_{1}, m_{2}}\left(u^{-1}\right)=1 \quad \text { for all } u \in U_{w}(\mathbb{R})
$$

holds, we define the Kloosterman sums

$$
S_{w}\left(\psi_{n_{1}, n_{2}}, \psi_{m_{1}, m_{2}} ; c\right)=\sum_{\gamma=b c w b^{\prime} \in U(\mathbb{Z}) \backslash \Gamma_{0}(N) / V \bar{U}_{w}(\mathbb{Z})} \psi_{n_{1}, n_{2}}(b) \psi_{m_{1}, m_{2}}\left(b^{\prime}\right) .
$$


The sum is over representatives $\gamma$ in the quotient space having the prescribed components $c$ and $w$ in their Bruhat decomposition, which is well-defined by the compatibility condition. The quotient by $V$ simply allows us to restrict to positive moduli $c_{1}$ and $c_{2}$ by conjugating the $v$ matrix, which contains the signs of the moduli, to the right. If the compatibility relation fails, we simply define $S_{w}\left(\psi_{n_{1}, n_{2}}, \psi_{m_{1}, m_{2}} ; c\right)=0$.

By a computation of Friedberg [Fr, pp. 173-174], only sums satisfying the compatibility condition occur in the Fourier expansion of a Poincaré series. In particular, for $n_{1} n_{2} m_{1} m_{2} \neq 0$, only the $I, w_{4}, w_{5}$, and $w_{6}$ Weyl elements contribute, since otherwise the compatibility relation is never satisfied.

We now wish to show that the concrete expressions for the Kloosterman sums given in (2.2) and (2.3) match the abstract definition (2.12).

We may parameterize representatives of $U(\mathbb{Z}) \backslash \Gamma_{0}(N)$ by the Plücker coordinates $A_{1}, B_{1}, C_{1}$ and $A_{2}, B_{2}, C_{2}$ satisfying

$$
\begin{aligned}
& \left(A_{1}, B_{1}, C_{1}\right)=\left(A_{2}, B_{2}, C_{2}\right)=1, \\
& A_{1} C_{2}+B_{1} B_{2}+C_{1} A_{2}=0, \\
& N\left|A_{1}, N\right| B_{1} .
\end{aligned}
$$

For a matrix $\gamma=\left(\begin{array}{lll}g & h & i \\ d & e & f \\ a & b & c\end{array}\right) \in \Gamma_{0}(N)$, these are computed by

$$
A_{1}=a, \quad B_{1}=b, \quad C_{1}=c, \quad A_{2}=b d-a e, \quad B_{2}=a f-c d, \quad C_{2}=c e-b f .
$$

Our computation now essentially follows $[\mathrm{BFG}]$, but we must keep track of the level condition $N\left|A_{1}, N\right|$ $B_{1}$. The auxiliary parameters $Z_{2}=g, Y_{2}=h, X_{2}=i, Z_{1}=g e-d h, Y_{1}=d i-g f$, and $X_{1}=f h-e i$ are solutions to the equations

$$
\begin{aligned}
& Z_{2} C_{2}+Y_{2} B_{2}+X_{2} A_{2}=1 \\
& Z_{1} C_{1}+Y_{1} B_{1}+X_{1} A_{1}=1
\end{aligned}
$$

These equations do not completely determine the auxiliary parameters, but we will only require that the auxiliary parameters are some solution, as the final expression for the Kloosterman sum will be independent of the choice. The right-translation action of $x \in U(\mathbb{Z})$ on the Plücker coordinates gives the new values

$$
\begin{aligned}
& \left(A_{1}, B_{1}, C_{1}\right) \mapsto\left(A_{1}, B_{1}+x_{2} A_{1}, C_{1}+x_{1} B_{1}+x_{3} A_{1}\right), \\
& \left(A_{2}, B_{2}, C_{2}\right) \mapsto\left(A_{2}, B_{2}-x_{1} A_{2}, C_{2}-x_{2} B_{2}+\left(x_{1} x_{2}-x_{3}\right) A_{2}\right) .
\end{aligned}
$$

Now the Bruhat decomposition for elements of the long element Weyl cell in $\Gamma_{0}(N)$ may be written as

$$
\gamma=\left(\begin{array}{ccc}
1 & \frac{Z_{2} B_{1}-Y_{2} A_{1}}{A_{2}} & \frac{Z_{2}}{A_{1}} \\
& 1 & \frac{Y_{1} A_{2}-Z_{1} B_{2}}{A_{1}} \\
& & 1
\end{array}\right)\left(\begin{array}{ccc}
\frac{1}{A_{2}} & & \\
& \frac{A_{2}}{A_{1}} & \\
& & A_{1}
\end{array}\right) w_{6}\left(\begin{array}{ccc}
1 & \frac{B_{1}}{A_{1}} & \frac{C_{1}}{A_{1}} \\
& 1 & -\frac{B_{2}}{A_{2}} \\
& & 1
\end{array}\right) .
$$

Note that we put our decompositions in the form $b c w b^{\prime}$ with $b^{\prime} \in \bar{U}_{w}(\mathbb{Q})$, as opposed to $[\mathrm{BFG}$, who put their decompositions in the form $b w c b^{\prime}$ with $b \in \bar{U}_{w^{-1}}(\mathbb{Q})$. The decompositons are equivalent, but the former is more standardized.

Restricting to a fundamental domain for the action of $\bar{U}_{w_{6}}(\mathbb{Z})=U(\mathbb{Z})$, we may write the sum (2.12) for $w=w_{6}$ as

$$
S_{w_{6}}\left(\psi_{n_{1}, n_{2}}, \psi_{m_{1}, m_{2}} ;\left(A_{1}, A_{2}\right)\right)=\sum_{B_{1}, C_{1}, B_{2}, C_{2}} e\left(n_{1} \frac{Y_{1} A_{2}-Z_{1} B_{2}}{A_{1}}+n_{2} \frac{Z_{2} B_{1}-Y_{2} A_{1}}{A_{2}}-m_{1} \frac{B_{2}}{A_{2}}+m_{2} \frac{B_{1}}{A_{1}}\right),
$$

where the sum is taken over $0 \leq B_{1}, C_{1}<A_{1}$ and $0 \leq B_{2}<A_{2}$ subject to (2.13) and (2.14). Now [BFG, Lemma 4.1] shows that the summand is independent of the choice of auxiliary parameters. Note that the compatibility condition is trivially true for the long element as $U_{w_{6}}(\mathbb{R})=\{I\}$. 
We conclude the long element analysis by mentioning that the proofs of [BFG, Lemmas 4.1, 4.2] show that the sum is well-defined if we replace the summation conditions with their modular equivalents

$$
\begin{aligned}
& B_{1}, C_{1} \quad\left(\bmod A_{1}\right), \quad B_{2} \quad\left(\bmod A_{2}\right), \quad N\left|A_{1}, \quad N\right| B_{1} \\
& A_{1} C_{2}+B_{1} B_{2}+C_{1} A_{2} \equiv 0 \quad\left(\bmod A_{1} A_{2}\right), \\
& \left(A_{1}, B_{1}, C_{1}\right)=1, \quad\left(A_{2}, B_{2}, C_{2}\right)=1, \\
& Z_{2} C_{2}+Y_{2} B_{2}=1 \quad\left(\bmod A_{2}\right), \quad Z_{1} C_{1}+Y_{1} B_{1}=1 \quad\left(\bmod A_{1}\right),
\end{aligned}
$$

and the sum is empty unless $N \mid A_{2}$. This matches the previous definition with

$$
S_{w_{6}}\left(\psi_{n_{1}, n_{2}}, \psi_{m_{1}, m_{2}} ;\left(A_{1}, A_{2}\right)\right)=\delta_{N \mid A_{1}} S^{(N)}\left(m_{2},-m_{1}, n_{1},-n_{2} ; A_{1}, A_{2}\right) .
$$

As noted in the proof of [BFG, Theorem 5.1], replacing $m_{1}, n_{2}, B_{2}, C_{2}, C_{1}, Y_{2}, Z_{2}, Z_{1}$ by their negatives leaves the sum invariant, so we may drop the negatives on $m_{1}$ and $n_{2}$.

Elements of the $w_{5}$ cell necessarily have $A_{1}=0$ and $B_{1}, A_{2} \neq 0$, so that $B_{1} \mid A_{2}$. With the Plücker and auxiliary coordinates as before, for $\gamma$ having $A_{1}=0$ we have

$$
\gamma=\left(\begin{array}{ccc}
1 & \frac{Z_{2} B_{1}}{A_{2}} & \frac{Y_{2}}{B_{1}} \\
& 1 & \frac{Z_{1} C_{2}-X_{1} A_{2}}{B_{1}} \\
& & 1
\end{array}\right)\left(\begin{array}{ccc}
\frac{1}{A_{2}} & & \\
& \frac{A_{2}}{B_{1}} & \\
& & B_{1}
\end{array}\right) w_{5}\left(\begin{array}{ccc}
1 & 0 & -\frac{C_{2}}{A_{2}} \\
& 1 & \frac{C_{1}}{B_{1}} \\
& & 1
\end{array}\right)
$$

The compatibility condition becomes $n_{1} A_{2}=m_{2} B_{1}^{2}$. Now the conditions (2.13) and (2.14) simplify to

$$
\begin{aligned}
& \left(B_{1}, C_{1}\right)=1, \quad\left(A_{2} / B_{1}, C_{2}\right)=1, \quad B_{2}=-C_{1} \frac{A_{2}}{B_{1}}, \quad N \mid B_{1}, \\
& Z_{1} C_{1} \equiv 1 \quad\left(\bmod B_{1}\right), \quad Z_{2} C_{2} \equiv 1 \quad\left(\bmod A_{2} / B_{1}\right),
\end{aligned}
$$

and the space $\bar{U}_{w_{5}}(\mathbb{Z}) \subset U(\mathbb{Z})$ is defined by $x_{2}=0$, so we may write the Kloosterman sum as

$$
S_{w_{5}}\left(\psi_{n_{1}, n_{2}}, \psi_{m_{1}, m_{2}} ;\left(B_{1}, A_{2}\right)\right)=\underset{\substack{n_{1} A_{2}=m_{2} B_{1}^{2} \\ N\left|B_{1}\right| A_{2}}}{S_{S}}\left(m_{1}, n_{1}, n_{2} ; B_{1}, A_{2}\right) .
$$

Unlike the long element case, no extra work is needed to justify our use of the modular summation conditions.

For the $w_{4}$ cell, we have $A_{2}=0$ and $A_{1}, B_{2} \neq 0$, so that $B_{2} \mid A_{1}$, and the compatibility condition is $n_{2} A_{1}=m_{1} B_{2}^{2}$. With the Plücker and auxiliary coordinates as before, we have

$$
\gamma=\left(\begin{array}{ccc}
1 & \frac{X_{2} A_{1}-Z_{2} C_{1}}{B_{2}} & \frac{Z_{2}}{A_{1}} \\
& 1 & -\frac{Z_{1} B_{2}}{A_{1}}
\end{array}\right)\left(\begin{array}{ccc}
\frac{1}{B_{2}} & & \\
& \frac{B_{2}}{A_{1}} & \\
& & 1
\end{array}\right) w_{4}\left(\begin{array}{ccc}
1 & -\frac{C_{2}}{B_{2}} & \frac{C_{1}}{A_{1}} \\
& 1 & 0 \\
& & 1
\end{array}\right) .
$$

Now the conditions (2.13) and (2.14) simplify to

$$
\begin{aligned}
& \left(A_{1} / B_{2}, C_{1}\right)=1, \quad\left(B_{2}, C_{2}\right)=1, \quad B_{1}=-C_{2} \frac{A_{1}}{B_{2}}, \quad N\left|A_{1}, \quad N\right| B_{1}, \\
& Z_{1} C_{1} \equiv 1 \quad\left(\bmod A_{1} / B_{2}\right), \quad Z_{2} C_{2} \equiv 1 \quad\left(\bmod B_{2}\right),
\end{aligned}
$$

but we may take this one step further: we have $B_{2} \frac{B_{1}}{N}=-C_{2} \frac{A_{1}}{N}$ and the condition $\left(B_{2}, C_{2}\right)=1$ implies $B_{2} \mid \frac{A_{1}}{N}$. Conversely, the condition $N B_{2} \mid A_{1}$ implies $N \mid\left(-C_{1} \frac{A_{1}}{B_{2}}\right)=B_{1}$, so we may write the Kloosterman sum as

$$
S_{w_{4}}\left(\psi_{n_{1}, n_{2}}, \psi_{m_{1}, m_{2}} ;\left(A_{1}, B_{2}\right)\right)=\underset{\substack{n_{2} A_{1}=m_{1} B_{2}^{2} \\ N B_{2} \mid A_{1}}}{\delta_{S}}\left(-m_{2},-n_{2},-n_{1} ; B_{2}, A_{1}\right)
$$

Note that changing the sign of both $n_{1}$ and $n_{2}$ leaves $\tilde{S}$ invariant by $C_{2} \mapsto-C_{2}$. 


\section{Weight FunCtions in the KuZnetsov formula}

In order to use the Kuznetsov formula for a spectral average, we need a function $F$ such that $\left|\left\langle F, \tilde{W}_{\mu}\right\rangle\right|^{2}$ appearing on the left hand side of (2.10) is bounded away from zero for $\mu \in \Omega \subseteq \mathfrak{a}_{\mathbb{C}}^{*}$. For our purposes the following slightly weaker statement suffices.

Lemma 1. For a fixed compact $\Omega \subseteq \mathfrak{a}_{\mathbb{C}}^{*}$ there is a finite collection of smooth compactly supported functions $F_{1}, \ldots, F_{J}$ such that $\sum_{j}\left|\left\langle F_{j}, \tilde{W}_{\mu}\right\rangle\right|^{2} \gg 1$ for $\mu \in \Omega$.

Proof. This follows from a simple compactness argument: for each $\mu \in \Omega$ choose an open set $S_{\mu} \subseteq \mathbb{R}_{>0}^{2}$ such that $\Re \tilde{W}_{\mu}(y) \neq 0$ for all $y \in S_{\mu}$ or $\Im \tilde{W}_{\mu}(y) \neq 0$ for all $y \in S_{\mu}$. This is possible by continuity of $\tilde{W}_{\mu}(y)$ in $y$. By continuity in $\mu$, we can choose open neighbourhoods $U_{\mu}$ about $\mu$ such that $\Re \tilde{W}_{\mu^{*}}(y) \neq 0$ for all $y \in S_{\mu}$ and all $\mu^{*} \in U_{\mu}$ or $\Im \tilde{W}_{\mu}(y) \neq 0$ for all $y \in S_{\mu}$ and all $\mu^{*} \in U_{\mu}$. By compactness we pick a finite collection of such neighbourhoods $U_{\mu_{1}}, \ldots, U_{\mu_{J}}$ covering $\Omega$, and define the corresponding $F_{j}$ to be real-valued functions with support on $S_{\mu_{j}}$ and non-vanishing on the interior ${\stackrel{S}{\mu_{j}}}_{\mu_{j}}$.

For the proof of Theorem 4 we will need a function that blows up on the exceptional spectrum. We recall that by unitarity the exceptional spectrum is parametrized by

$$
\mu=(\rho+i \gamma,-\rho+i \gamma,-2 i \gamma)
$$

for $\gamma \in \mathbb{R}, \rho \in[-1 / 2,1 / 2]$ (by the Jacquet-Shalika bounds) and its translates under the Weyl group. For a fixed smooth compactly supported function $F$ and two parameters $X_{1}, X_{2} \geqslant 1$ let

$$
F^{\left(X_{1}, X_{2}\right)}\left(y_{1}, y_{2}\right):=F\left(X_{1} y_{1}, X_{2} y_{2}\right)
$$

so that $F=F^{(1,1)}$.

Lemma 2. Fix $\Omega \subseteq \mathfrak{a}_{\mathbb{C}}^{*}$, and let $X_{1}, X_{2} \geqslant 1, \varepsilon>0$. Assume that $F$ is non-negative and supported in a (depending on $\Omega$ ) sufficiently small neighbourhood about $(1,1)$ and that $X_{1}, X_{2}$ are sufficiently large. Then for exceptional $\mu \in \Omega$ of the form (3.1) with $|\rho| \geqslant \varepsilon$ we have $\left\langle F^{\left(X_{1}, X_{2}\right)}, \tilde{W}_{\mu}\right\rangle \asymp\left(X_{1} X_{2}\right)^{1+|\rho|}$.

Proof. We have by (2.6) that

$$
\begin{aligned}
& \left\langle F^{\left(X_{1}, X_{2}\right)}, \tilde{W}_{\mu}\right\rangle \\
& =\int_{(1)} \int_{(1)} \frac{\cosh \left(\frac{3}{2} \pi \gamma\right) \prod_{j=1}^{3} \Gamma\left(\frac{1}{2}\left(s_{1}+\mu_{j}\right)\right) \prod_{j=1}^{3} \Gamma\left(\frac{1}{2}\left(s_{2}-\mu_{j}\right)\right)}{4 \pi^{s_{1}+s_{2}} \Gamma\left(\frac{1}{2}\left(s_{1}+s_{2}\right)\right)} \mathcal{F}\left(-1-s_{1},-1-s_{2}\right) X_{1}^{1+s_{1}} X_{2}^{1+s_{2}} \frac{d s_{1} d s_{2}}{(2 \pi i)^{2}}
\end{aligned}
$$

where $\mathcal{F}$ is the double Mellin transform of $F$, an entire function in both variables. If without loss of generality $\rho>0$ (note that in particular $\mu_{1}, \mu_{2}, \mu_{3}$ are sufficiently distinct), we shift contours to the left and obtain

$$
\left\langle F^{\left(X_{1}, X_{2}\right)}, \tilde{W}_{\mu}\right\rangle=c_{\mu} \mathcal{F}(-1-\rho+i \gamma,-1-\rho-i \gamma)\left(X_{1}^{1+\rho-i \gamma}+O\left(X_{1}\right)\right)\left(X_{2}^{1+\rho+i \gamma}+O\left(X_{2}\right)\right)
$$

for some constant $c_{\mu} \neq 0$. If $F$ is non-negative and supported in a sufficiently small neighbourhood about $(1,1)$, then $\mathcal{F}(-1-\rho+i \gamma,-1-\rho-i \gamma) \neq 0$ for all $\mu \in \Omega$. This proves the lemma.

Next we provide bounds for the functions $\tilde{\mathcal{J}}_{\epsilon ; F}(A)$ and $\mathcal{J}_{\epsilon ; F}\left(A_{1}, A_{2}\right)$ defined in $(2.4)$ and (2.5). Here $F$ will always be a fixed compactly supported function and all implied constants may depend on $F$. For bounds in the case of certain highly oscillating functions $F$ see [Bl, Proposition 5]. We define $F^{\left(X_{1}, X_{2}\right)}$ as in (3.2). The following basic bound suffices in many cases.

Lemma 3. Let $X_{1}, X_{2} \geqslant 1$.

(a) We have $\tilde{\mathcal{J}}_{\epsilon ; F^{\left(X_{1}, X_{2}\right)}}(A)=0$ unless $A \gg X_{1}^{-3 / 2}+X_{2}^{-3 / 2}$, in which case

$$
\tilde{\mathcal{J}}_{\epsilon ; F^{\left(X_{1}, X_{2}\right)}}(A) \ll\left(X_{1} X_{2}\right)^{2} .
$$


(b) We have $\mathcal{J}_{\epsilon ; F^{\left(X_{1}, X_{2}\right)}}\left(A_{1}, A_{2}\right)=0$ unless $\min \left(A_{1} A_{2}^{2}, A_{2} A_{1}^{2}\right) \gg\left(X_{1} X_{2}\right)^{-3 / 2}$, in which case

$$
\frac{d^{i}}{d A_{1}^{i}} \frac{d^{j}}{d A_{2}^{j}} \mathcal{J}_{\epsilon ; F^{\left(X_{1}, X_{2}\right)}}\left(A_{1}, A_{2}\right) \ll_{i, j}\left(X_{1} X_{2}\right)^{2}\left(A_{1} A_{2}\right)^{\varepsilon}\left(A_{2}^{2 / 3} A_{1}^{1 / 3}\right)^{i}\left(A_{1}^{2 / 3} A_{2}^{1 / 3}\right)^{j}
$$

for all $i, j \in \mathbb{N}_{0}=\{0,1,2, \ldots\}$.

Remark: Except for one instance in the proof of Theorem 4 we will always apply this lemma with $X_{1}=X_{2}=1$, so for most of the paper the variables $X_{1}, X_{2}$ can be ignored.

Proof. (a) This is straightforward from the definition and uses only trivial bounds, noting that the support of $F^{\left(X_{1}, X_{2}\right)}$ restricts the variables to

$$
y_{1} \asymp\left(X_{1} A\right)^{-1}, \quad y_{2} \asymp X_{2}^{-1}, \quad 1+x_{1}^{2} \asymp A^{4 / 3} X_{1}^{2}, \quad 1+x_{1}^{2}+x_{2}^{2} \asymp A^{8 / 3}\left(X_{1} X_{2}\right)^{2} .
$$

This forces $A \gg X_{1}^{-3 / 2}+X_{2}^{-3 / 2}$. The upper bound follows now from trivial estimates ${ }^{1}$.

(b) The support of $F^{\left(X_{1}, X_{2}\right)}$ restricts the variables to

$$
\begin{aligned}
& y_{1} \asymp\left(X_{1} A_{1}\right)^{-1}, \quad y_{2} \asymp\left(X_{2} A_{2}\right)^{-1}, \\
& \left(x_{1} x_{2}-x_{3}\right)^{2}+x_{1}^{2}+1=: \xi_{1} \asymp \Xi_{1}:=A_{2}^{4 / 3} A_{1}^{8 / 3}\left(X_{1} X_{2}\right)^{2}, \quad x_{3}^{2}+x_{2}^{2}+1=: \xi_{2} \asymp \Xi_{2}:=A_{1}^{4 / 3} A_{2}^{8 / 3}\left(X_{1} X_{2}\right)^{2}
\end{aligned}
$$

which implies that both $A_{1} A_{2}^{2}$ and $A_{1}^{2} A_{2}$ must be at least of order $\left(X_{1} X_{2}\right)^{-3 / 2}$. We recall from [Bl, Lemma 4] that

$$
\int_{\substack{\xi_{1} \asymp \Xi_{1} \\ \xi_{2} \asymp \Xi_{2}}} d x_{1} d x_{2} d x_{3} \ll\left(\Xi_{1} \Xi_{2}\right)^{1 / 2+\varepsilon}=\left(A_{1} A_{2} X_{1} X_{2}\right)^{2+\varepsilon}
$$

for $\Xi_{1}, \Xi_{2} \gg 1$. For the derivatives we differentiate under the integral sign and estimate trivially, see also $[\mathrm{Bl},(8.16)]$.

For one application we need a more refined estimate of a certain 6-fold Fourier transform involving $\mathcal{J}_{\epsilon ; F}$.

Lemma 4. Let $W:(0, \infty)^{6} \rightarrow \mathbb{C}$ be a fixed smooth compactly supported function. Let $A_{1}, A_{2}>0$ and define $A:=\exp \left(\max \left(\left|\log A_{1}\right|,\left|\log A_{2}\right|\right)\right)$. Let $P \geqslant 1$, and let $\alpha_{1}, \alpha_{2}, \beta_{1}, \beta_{2}, \gamma_{1}, \gamma_{2} \in \mathbb{R}$ be such that $\min \left(\left|\alpha_{1}\right|,\left|\alpha_{2}\right|,\left|\beta_{1}\right|,\left|\beta_{2}\right|,\left|\gamma_{1}\right|,\left|\gamma_{2}\right|\right) \leqslant P$. Then the six-fold Fourier transform

$$
\begin{aligned}
\widehat{\mathcal{J}}:=\int_{\mathbb{R}^{6}} \mathcal{J}_{\epsilon ; F} & \left(A_{1} \sqrt{t_{1} u_{1} v_{1}}, A_{2} \sqrt{t_{2} u_{2} v_{2}}\right) W\left(t_{1}, t_{2}, u_{1}, u_{2}, v_{1}, v_{2}\right) \\
& \times e\left(-t_{1} \alpha_{1}-t_{2} \alpha_{2}-u_{1} \beta_{1}-u_{2} \beta_{2}-v_{1} \gamma_{1}-v_{2} \gamma_{2}\right) d t_{1} d t_{2} d u_{1} d u_{2} d v_{1} d v_{2}
\end{aligned}
$$

is bounded by

$$
O_{C}\left((P A)^{\varepsilon}\left(P^{2} \max \left(A_{2}^{-2 / 3} A_{1}^{-4 / 3}, A_{1}^{-2 / 3} A_{2}^{-4 / 3}\right)+P^{-C}\right)\right)
$$

for any constant $C>0$. In addition, it is bounded by

$$
A^{\varepsilon} \max \left(\left|\alpha_{1}\right|,\left|\beta_{1}\right|,\left|\gamma_{1}\right|\right)^{-1 / 2} \max \left(\left|\alpha_{2}\right|,\left|\beta_{2}\right|,\left|\gamma_{2}\right|\right)^{-1 / 2},
$$

as long as both maxima are non-zero.

Proof. We recall the notation

$$
\left(x_{1} x_{2}-x_{3}\right)^{2}+x_{1}^{2}+1=: \xi_{1} \asymp \Xi_{1}:=A_{2}^{4 / 3} A_{1}^{8 / 3}, \quad x_{3}^{2}+x_{2}^{2}+1=: \xi_{2} \asymp \Xi_{2}:=A_{1}^{4 / 3} A_{2}^{8 / 3}
$$

from the previous proof. We will frequently use (3.3) with $X_{1}=X_{2}=1$. We also write $x_{4}=x_{1} x_{2}-x_{3}$ and

$$
\eta_{1}=x_{2} x_{4}+x_{1}, \quad \eta_{2}=x_{1} x_{3}+x_{2}
$$

\footnotetext{
${ }^{1}$ This corrects an error $[\mathrm{Bl},(8.7)]$ where $X_{1}^{2} X_{2}$ should be replaced with $X_{1}^{2} X_{2}^{2}$.
} 
We express $\mathcal{J}_{\epsilon ; F}$ by its defining 5 -fold integral (2.5) and write

$$
\mathcal{J}_{\epsilon ; F}\left(A_{1}, A_{2}\right)=\frac{1}{\left(A_{1} A_{2}\right)^{2}} \int_{\substack{\xi_{1} \asymp \Xi_{1} \asymp \Xi_{2} \\ \xi_{2}}} \mathcal{K}\left(A_{1}, A_{2} ; x_{1}, x_{2}, x_{3}\right) d x_{1} d x_{2} d x_{3}
$$

where $\mathcal{K}$ is the double $y_{1}, y_{2}$-integral, i.e.

$$
\begin{aligned}
\mathcal{K}\left(A_{1}, A_{2} ; x_{1}, x_{2}, x_{3}\right)=\int_{0}^{\infty} \int_{0}^{\infty} & e\left(-\epsilon_{1} A_{1} x_{1} y_{1}-\epsilon_{2} A_{2} x_{2} y_{2}-\frac{A_{2} \eta_{2}}{y_{2} \xi_{2}}-\frac{A_{1} \eta_{1}}{y_{1} \xi_{1}}\right) \\
& \times \overline{F\left(A_{1} y_{1}, A_{2} y_{2}\right)} F\left(\frac{A_{2} \xi_{1}^{1 / 2}}{y_{2} \xi_{2}}, \frac{A_{1} \xi_{2}^{1 / 2}}{y_{1} \xi_{1}}\right) \frac{d y_{1} d y_{2}}{y_{1} y_{2}} .
\end{aligned}
$$

We start with the proof of (3.4). Suppose that $\left|\alpha_{1}\right|$ is the smallest of the variables (possibly $\left|\alpha_{1}\right|=0$ ). Choose a sufficiently large constant $c_{2}$ and a sufficiently large constant $c_{1}>c_{2}$. We split the $x_{1}, x_{2}, x_{3^{-}}$ integration in four pieces

$$
\text { (i) }\left|x_{1}\right|, A_{1}^{2}\left|\eta_{1}\right| / \xi_{1} \leqslant c_{1} P, \quad \text { (ii) }\left|x_{1}\right| \leqslant c_{2} P, A_{1}^{2}\left|\eta_{1}\right| / \xi_{1} \geqslant c_{1} P, \quad \text { (iii) }\left|x_{1}\right| \geqslant c_{1} P, A_{1}^{2}\left|\eta_{1}\right| / \xi_{1} \leqslant c_{2} P
$$

and the remaining portion (iv), which is contained in $\left|x_{1}\right|, A_{1}^{2}\left|\eta_{1}\right| / \xi_{1} \geqslant c_{2} P$. The conditions (i) imply $\left|x_{1}\right| \ll P, x_{2} x_{4} \ll P A_{2}^{4 / 3} A_{1}^{2 / 3}$ (note that we may assume by Lemma 3 that this is $\gg P$ ). The area of this region is

$$
\ll P \int_{\substack{x_{2} x_{4} \ll P A_{2}^{4 / 3} A_{1}^{2 / 3} \\ x_{2}, x_{4} \ll A^{O(1)}}} d x_{2} d x_{4} \ll P^{2} A_{2}^{4 / 3} A_{1}^{2 / 3}(A P)^{\varepsilon} .
$$

As $\mathcal{K}\left(A_{1}, A_{2} ; x_{1}, x_{2}, x_{3}\right) \ll 1$, the total contribution of this case to $\widehat{\mathcal{J}}$ is $P^{2} A_{2}^{-2 / 3} A_{1}^{-4 / 3}(A P)^{\varepsilon}$.

To deal with the region (ii), we note that the phase in the $y_{1}$-integral in (3.6) is given by

$$
e\left(-\epsilon_{1} A_{1} x_{1} y_{1}-\frac{A_{1} \eta_{1}}{y_{1} \xi_{1}}\right) .
$$

If $c_{1}$ is sufficiently large compared to $c_{2}$ (or if $\epsilon_{1} x_{1}$ and $\eta$ have different signs), the phase has no stationary point, and after sufficiently many integrations by parts, using for instance [BKY, Lemma 8.1] with

$$
X=A_{1}, \quad U=Q=\frac{1}{A_{1}}, \quad Y=P, \quad R=A_{1} Y
$$

we bound after trivial estimation in all other variables this portion of $\widehat{\mathcal{J}}$ by $\ll A^{\varepsilon} P^{-C}$. The same argument works for the region (iii).

In order to analyze the region (iv), we consider the expression (3.6) in more detail, first without any restrictions on the $x$-variables. We could run a careful stationary phase argument as in [BKY, Proposition 8.2], but we can also proceed in a completely elementary way. Applying the stationary phase method only on a formal basis shows that the oscillation of the $y_{1}$-integral is given by

$$
e\left(-\operatorname{sgn}\left(\eta_{1}\right) \frac{2 \sqrt{\left|x_{1} \eta_{1}\right|} A_{1}}{\sqrt{\xi_{1}}}\right)
$$

coming from the stationary point at $y_{1}=\left(\eta_{1} /\left(\epsilon_{1} x_{1} \xi_{1}\right)\right)^{1 / 2}$. With this in mind let us define

$$
\begin{aligned}
\tilde{\mathcal{K}}\left(A_{1}, A_{2} ; x_{1}, x_{2}, x_{3}\right) & :=e\left(\operatorname{sgn}\left(\eta_{1}\right) \frac{2 \sqrt{\left|x_{1} \eta_{1}\right|} A_{1}}{\sqrt{\xi_{1}}}+\operatorname{sgn}\left(\eta_{2}\right) \frac{2 \sqrt{\left|x_{2} \eta_{2}\right|} A_{2}}{\sqrt{\xi_{2}}}\right) \mathcal{K}\left(A_{1}, A_{2} ; x_{1}, x_{2}, x_{3}\right) \\
& =\int_{0}^{\infty} \int_{0}^{\infty} e\left(g\left(A_{1}, y_{1}\right)+h\left(A_{2}, y_{2}\right)\right) \overline{F\left(A_{1} y_{1}, A_{2} y_{2}\right)} F\left(\frac{A_{2} \xi_{1}^{1 / 2}}{y_{2} \xi_{2}}, \frac{A_{1} \xi_{2}^{1 / 2}}{y_{1} \xi_{1}}\right) \frac{d y_{1} d y_{2}}{y_{1} y_{2}}
\end{aligned}
$$

with

$$
g\left(A_{1}, y_{1}\right)=g_{\epsilon_{1} x_{1}, \eta_{1}, \xi_{1}}\left(A_{1}, y_{1}\right)=-\epsilon_{1} A_{1} x_{1} y_{1}-\frac{A_{1} \eta_{1}}{y_{1} \xi_{1}}+\operatorname{sgn}\left(\eta_{1}\right) \frac{2 \sqrt{\left|x_{1} \eta_{1}\right|} A_{1}}{\sqrt{\xi_{1}}}
$$


and

$$
h\left(A_{2}, y_{2}\right)=h_{\epsilon_{2} x_{2}, \eta_{2}, \xi_{2}}\left(A_{2}, y_{2}\right)=-\epsilon_{2} A_{2} x_{2} y_{2}-\frac{A_{2} \eta_{2}}{y_{2} \xi_{2}}+\operatorname{sgn}\left(\eta_{2}\right) \frac{2 \sqrt{\left|x_{2} \eta_{2}\right|} A_{2}}{\sqrt{\xi_{2}}} .
$$

We show the uniform bound

$$
\frac{\partial^{i}}{\partial A_{1}^{i}} \frac{\partial^{j}}{\partial A_{2}^{j}} \tilde{\mathcal{K}}\left(A_{1}, A_{2} ; x_{1}, x_{2}, x_{3}\right) \ll_{i, j} A_{1}^{-i} A_{2}^{-j} .
$$

Indeed, one checks by direct computation that

$$
\frac{\frac{\partial}{\partial A_{1}} g\left(A_{1}, y_{1}\right)}{\frac{\partial}{\partial y_{1}} g\left(A_{1}, y_{1}\right)}= \pm \frac{y_{1}}{A_{1}} \cdot \frac{\sqrt{\left|\eta_{1}\right|}-\sqrt{\left|x_{1}\right| \xi_{1}} y_{1}}{\sqrt{\left|\eta_{1}\right|}+\sqrt{\left|x_{1}\right| \xi_{1}} y_{1}}
$$

so that

$$
\frac{\partial^{i}}{\partial y_{1}^{i}} \frac{\partial^{j}}{\partial A_{1}^{j}}\left(\frac{\frac{\partial}{\partial A_{1}} g\left(A_{1}, y_{1}\right)}{\frac{\partial}{\partial y_{1}} g\left(A_{1}, y_{1}\right)}\right) \ll_{i, j} \frac{y_{1}}{A_{1}} y_{1}^{-i} A_{1}^{-j}
$$

for $i, j \in \mathbb{N}_{0}$. Hence combining each differentiation with respect to $A_{1}$ with an integration by parts in $y_{1}$, we obtain the desired bound (3.7) in $A_{1}$, and the bound in $A_{2}$ follows similarly.

Having proved (3.7), we return to the estimation of $\widehat{\mathcal{J}}$. The phase of the Fourier integral in question is given by

$$
e\left( \pm \frac{2 \sqrt{\left|x_{1} \eta_{1}\right|} A_{1} \sqrt{t_{1} u_{1} v_{1}}}{\sqrt{\xi_{1}}} \pm \frac{2 \sqrt{\left|x_{2} \eta_{2}\right|} A_{2} \sqrt{t_{2} u_{2} v_{2}}}{\sqrt{\xi_{2}}}-t_{1} \alpha_{1}-t_{2} \alpha_{2}-u_{1} \beta_{1}-u_{2} \beta_{2}-v_{1} \gamma_{1}-v_{2} \gamma_{2}\right)
$$

which needs to be integrated against the non-oscillating functions

$$
\tilde{\mathcal{K}}\left(A_{1} \sqrt{t_{1} u_{1} v_{1}}, A_{2} \sqrt{t_{2} u_{2} v_{2}} ; x_{1}, x_{2}, x_{3}\right) W\left(t_{1}, t_{2}, u_{1}, u_{2}, v_{1}, v_{2}\right)
$$

with respect to $x_{1}, x_{2}, x_{3}$ and $t_{1}, t_{2}, u_{1}, u_{2}, v_{1}, v_{2}$. If we are in the region (iv), then in particular $\left|x_{1}\right|, A_{1}^{2}\left|\eta_{1}\right| / \xi_{1} \geqslant$ $c_{2} P$, as mentioned above. Since $\left|\alpha_{1}\right| \leqslant P$, the phase has no stationary point if $c_{2}$ is sufficiently large, and by repeated partial integration in any of the variables $t_{1}$ we obtain again the bound $A^{\varepsilon} P^{-C}$. This completes the proof of (3.4) if $\left|\alpha_{1}\right|$ is minimal. If any of the other variables is minimal, we can run the same argument, possibly with interchanged indices.

For the bound (3.5) we return to (3.8) for an arbitrary choice of $x_{1}, x_{2}, x_{3}$. The simple stationary phase type bound

$$
\int e(a t+b \sqrt{t}) W(t) d t \ll|a|^{-1 / 2}, \quad a \neq 0
$$

for a fixed smooth function $W$ with compact support in $(0, \infty)$ applied twice, followed by trivial estimations, yields readily the bound (3.5). This completes the proof.

\section{Kloosterman SUmS}

In this section we collect some results about the Kloosterman sums defined in (2.2) and (2.3). We start with useful upper bounds.

Lemma 5. Let $N, D_{1}, D_{2} \in \mathbb{N}, m_{1}, m_{2}, n_{1}, n_{2} \in \mathbb{Z} \backslash\{0\}$. We have

$$
\tilde{S}\left(m_{1}, m_{2}, n_{1} ; D_{1}, D_{2}\right) \ll\left(\left(n_{2}, D_{2} / D_{1}\right) D_{1}^{2},\left(m_{1}, n_{1}, D_{1}\right) D_{2}\right)\left(D_{1} D_{2}\right)^{\varepsilon}
$$

and

$$
S^{(N)}\left(m_{1}, m_{2}, n_{1}, n_{2} ; D_{1}, D_{2}\right) \ll\left(D_{1} D_{2}\right)^{1 / 2+\varepsilon}\left(\left(D_{1}, D_{2}\right)\left(m_{1} n_{1},\left[D_{1}, D_{2}\right]\right)\left(m_{2} n_{2},\left[D_{1}, D_{2}\right]\right)\right)^{1 / 2}
$$

for any $\varepsilon>0$. 
Proof. The bound for $\tilde{S}$ is Larsen's bound [BFG, Appendix]. The bound for $S^{(N)}$ is Stevens' bound [St, Theorem 5.1] in its uniform version given in [Bu, p. 39]. Note that for the level $N$ Kloosterman sum, only those $S_{a, b}\left(n, \psi, \psi^{\prime}\right)$ (in the notation of [St, Section 5]) contribute to the Kloosterman sum where $a \leqslant s-k$, $b \leqslant r$ with $s, r \geqslant k$ whenever $p^{k} \| N$ (cf. also [DF, Remark 2.5]). In particular, Stevens' bound holds a fortiori for level $N$ Kloosterman sums.

As in $[\mathrm{Bl} \text {, Lemma } 3]^{2}$ we conclude from (4.1) that

$$
\begin{aligned}
& \sum_{\substack{N\left|D_{1} \leqslant X_{1} \\
N\right| D_{2} \leqslant X_{2}}}\left|S^{(N)}\left(m_{1}, m_{2}, n_{1}, n_{2} ; D_{1}, D_{2}\right)\right| \\
& \ll\left(X_{1} X_{2}\right)^{1 / 2+\varepsilon} N^{1 / 2} \sum_{\substack{\delta \delta_{1} \leqslant X_{1} / N \\
\delta \delta_{2} \leqslant X_{2} / N}} \delta^{1 / 2}\left(\left(m_{1} n_{1}, \delta_{1}\right)\left(m_{1} n_{1}, \delta_{2}\right)\left(m_{1} n_{2}, \delta\right)\left(m_{2} n_{2}, \delta_{1}\right)\left(m_{2} n_{2}, \delta_{2}\right)\left(m_{2} n_{2}, \delta\right)\right)^{1 / 2} \\
& \ll \frac{\left(X_{1} X_{2}\right)^{3 / 2+\varepsilon}\left(m_{1} n_{2} m_{2} n_{1}\right)^{\varepsilon}}{N^{3 / 2}} \sum_{\delta \leqslant X_{1}} \frac{\left(m_{1} n_{1}, \delta\right)^{1 / 2}\left(m_{2} n_{2}, \delta\right)^{1 / 2}}{\delta^{3 / 2}} \ll \frac{\left(X_{1} X_{2}\right)^{3 / 2+\varepsilon}\left(m_{1} n_{2} m_{2} n_{1}\right)^{\varepsilon}}{N^{3 / 2}}
\end{aligned}
$$

if $\left(m_{1} m_{2} n_{1} n_{2}, N\right)=1$.

Lemma 6. For $N \in \mathbb{N}$ the following holds.

(a) The sum $S^{(N)}\left(m_{1}, m_{2}, n_{1}, n_{2} ; D_{1}, D_{2}\right)$ depends only on $m_{j}, n_{j}$ modulo $D_{j}$ for $j=1,2$.

(b) If $\left(t_{1} t_{2}, u_{1} u_{2}\right)=1$ for $j=1,2$ and $N \mid t_{1} u_{1}, t_{2} u_{2}$, then

$$
\begin{aligned}
& S^{(N)}\left(m_{1}, m_{2}, n_{1}, n_{2} ; t_{1} u_{1}, t_{2} u_{2}\right) \\
& =S^{\left(\operatorname{gcd}\left(N, t_{1}\right)\right)}\left(\bar{u}_{1}^{2} u_{2} m_{1}, \bar{u}_{2}^{2} u_{1} m_{2}, n_{1}, n_{2} ; t_{1}, t_{2}\right) S^{\left(\operatorname{gcd}\left(N, u_{1}\right)\right)}\left(\vec{t}_{1}^{2} t_{2} m_{1}, \vec{t}_{2}^{2} t_{1} m_{2}, n_{1}, n_{2} ; u_{1}, u_{2}\right) .
\end{aligned}
$$

(c) Let $N$ be prime and let $r_{q}(n)$ denote the Ramanujan sum. Then

$$
S^{(N)}\left(m_{1}, m_{2}, n_{1}, n_{2} ; N, N\right)=N-1+r_{N}\left(n_{1}\right) r_{N}\left(m_{2}\right)= \begin{cases}N(N-1), & N\left|n_{1}, N\right| m_{2}, \\ N, & N \nmid n_{1} m_{2}, \\ 0 & \text { else. }\end{cases}
$$

Proof. This is proved as in [BFG, Section 4], cf. Properties 4.6, 4.7 and 4.10, respectively. Part (a) is trivial.

For part (b) we observe that the assumptions $\left(t_{1}, u_{1}\right)=1$ and $N \mid t_{1} u_{1}$ imply that

$$
\left(\left(N, t_{1}\right) u_{1},\left(N, u_{1}\right) t_{1}\right)=N \text {. }
$$

We now follow verbatim the proof of [BFG, Property 4.7]. Given two sets of summation variables $B_{j}, C_{j}$ and $B_{j}^{\prime}, C_{j}^{\prime}$ such that $\left(B_{j}, C_{j}, t_{j}\right)=\left(B_{j}^{\prime}, C_{j}^{\prime}, u_{j}\right)=1$ for $j=1,2,\left(N, t_{1}\right)\left|B_{1},\left(N, u_{1}\right)\right| B_{1}^{\prime}, t_{1} t_{2} \mid t_{1} C_{2}+$ $B_{1} B_{2}+C_{1} t_{2}$ and $u_{1} u_{2} \mid u_{1} C_{2}^{\prime}+B_{1}^{\prime} B_{2}^{\prime}+C_{1}^{\prime} u_{2}$ we choose $r, r^{\prime} \in \mathbb{Z}$ with $r t_{1} t_{2}+r^{\prime} u_{1} u_{2}=1$. We define new variables

$$
\begin{array}{lll}
d_{1}=t_{1} u_{1}, & b_{1}=r^{\prime} u_{1} u_{2} B_{1}+r t_{1} t_{2} B_{1}^{\prime}, & c_{1}=\left(r^{\prime}\right)^{2} u_{1}^{2} u_{2} C_{1}+r^{2} t_{1}^{2} t_{2} C_{1}^{\prime}, \\
d_{2}=t_{2} u_{2}, & b_{2}=r^{\prime} u_{1} u_{2} B_{2}+r t_{1} t_{2} B_{2}^{\prime}, & c_{2}=\left(r^{\prime}\right)^{2} u_{1} u_{2}^{2} C_{2}+r^{2} t_{1} t_{2}^{2} C_{2}^{\prime},
\end{array}
$$

and observe that $b_{1}$ runs through all numbers modulo $d_{1}=t_{1} u_{1}$ that are divisible by (4.3), which is the desired extra divisibility condition $N \mid b_{1}$ for $S^{(N)}\left(m_{1}, m_{2}, n_{1}, n_{2} ; t_{1} u_{1}, t_{2} u_{2}\right)$. Now we continue verbatim

\footnotetext{
${ }^{2}$ Notice that in [Bl, Lemma 3] the indices should be exchanged and read as in (4.1) above as a consequence of Remark 1 after Theorem 6 .
} 
as in $[\mathrm{BFG}]$.

To prove (c) we observe that $S^{(N)}\left(m_{1}, m_{2}, n_{1}, n_{2} ; N, N\right)$ for $N$ prime equals

$$
\begin{aligned}
& \sum_{\substack{C_{1}, B_{2}, C_{2}(\bmod N) \\
C_{2}+C_{1} \equiv 0(\bmod N) \\
\left(C_{1}, N\right)=\left(B_{2}, C_{2}, N\right)=1}} e\left(\frac{-n_{1} \bar{C}_{1} B_{2}}{N}+\frac{m_{2} B_{2}}{N}\right)=\sum_{\substack{B, C(\bmod N) \\
(C, N)=1}} e\left(\frac{-n_{1} C B}{N}+\frac{m_{2} B}{N}\right) \\
& =N-1+\sum_{\substack{B, C(\bmod N) \\
(B C, N)=1}} e\left(\frac{-n_{1} C B}{N}+\frac{m_{2} B}{N}\right)=N-1+r_{N}\left(n_{1}\right) r_{N}\left(m_{2}\right) .
\end{aligned}
$$

This completes the proof of the lemma.

Remark: For completeness we also state the following two properties of the level $N$ Kloosterman sums that can be proved as in Property 4.3 and Property $4.4+4.5$ of [BFG]:

(d) For $\left(D_{1} D_{2}, a b\right)=1$ we have $S^{(N)}\left(a m_{1}, b m_{2}, n_{1}, n_{2} ; D_{1}, D_{2}\right)=S^{(N)}\left(m_{1}, m_{2}, a n_{1}, b n_{2} ; D_{1}, D_{2}\right)$.

(e) We have $S^{(N)}\left(m_{1}, m_{2}, n_{1}, n_{2} ; D_{1}, D_{2}\right)=S^{(N)}\left(n_{2}, n_{1}, m_{2}, m_{1} ; D_{2}, D_{1}\right)$.

Note, however, that an analogue of Property 4.4 or Property 4.5 alone does not exist due to asymmetry of the summation condition $N \mid B_{1}$. We do not need the statements (d) and (e) in this paper.

For later purposes we study a certain 6-fold Fourier transform of the long Weyl element Kloosterman sum. Let $d, D_{1}, D_{2} \in \mathbb{N}$, and let $a \in\left(\mathbb{Z} / D_{1} Z\right)^{*}$ and $b \in\left(\mathbb{Z} / D_{2} \mathbb{Z}\right)^{*}$. For integers $x_{1}, x_{2}, y_{1}, y_{2}, z_{1}, z_{2}$ we define

$$
\begin{aligned}
\widehat{S}_{a, b, d}\left(x_{1}, x_{2}, y_{1}, y_{2}, z_{1}, z_{2} ; D_{1}, D_{2}\right):= & \frac{1}{D_{1}^{3} D_{2}^{3}} \sum_{n_{1}, m_{1}, l_{1}\left(\bmod D_{1}\right)} S^{(1)}\left(a m_{1} d, b n_{2} l_{2}, n_{1} l_{1}, m_{2} d ; D_{1}, D_{2}\right) \\
& \times e\left(-\frac{n_{1} x_{1}+m_{1} y_{1}+l_{1} z_{1}}{n_{1}}\right) e\left(-\frac{n_{2} x_{2}+m_{2} y_{2}+l_{2} z_{2}}{D_{2}}\right) .
\end{aligned}
$$

This is the non-archimedean analogue of the function studied in Lemma 4.

We also need a twisted version. Let $\chi$ be a primitive character modulo a prime $p$ such that $(d, p)=1$. Assume that $p^{3} \mid D_{1}$ and $p^{3} \mid D_{2}$. Then we define

$$
\begin{aligned}
& \widehat{S}_{a, b, d}^{\chi}\left(x_{1}, x_{2}, y_{1}, y_{2}, z_{1}, z_{2} ; D_{1}, D_{2}\right):=\frac{1}{D_{1}^{3} D_{2}^{3}} \sum_{\substack{n_{1}, m_{1}, l_{1}\left(\bmod D_{1}\right) \\
n_{2}, m_{2}, l_{2}\left(\bmod D_{2}\right)}} \bar{\chi}\left(n_{1} l_{1} m_{2}\right) \chi\left(m_{1} n_{2} l_{2}\right) \\
& \quad \times S^{\left(p^{3}\right)}\left(a m_{1} d, b n_{2} l_{2}, n_{1} l_{1}, m_{2} d ; D_{1}, D_{2}\right) e\left(-\frac{n_{1} x_{1}+m_{1} y_{1}+l_{1} z_{1}}{D_{1}}\right) e\left(-\frac{n_{2} x_{2}+m_{2} y_{2}+l_{2} z_{2}}{D_{2}}\right) .
\end{aligned}
$$

By the Chinese remainder theorem and Lemma 6(b) we have the following multiplicativity formulae

$$
\begin{aligned}
\widehat{S}_{a, b, d}^{\chi}\left(x_{1}, x_{2}, y_{1}, y_{2}, z_{1}, z_{2} ; t_{1} p^{\alpha_{1}}, t_{2} p^{\alpha_{2}}\right) & =\widehat{S}_{a, b, d}^{\chi}\left(\bar{t}_{1} x_{1}, t_{2} \bar{t}_{1} x_{2}, t_{1} \bar{t}_{2} y_{1}, \bar{t}_{2} y_{2}, \bar{t}_{1} z_{1}, \bar{t}_{2} z_{2} ; p^{\alpha_{1}}, p^{\alpha_{2}}\right) \\
& \times \widehat{S}_{a, b, d}\left(\overline{p^{\alpha_{1}}} x_{1}, p^{\alpha_{2}} \overline{p^{\alpha_{1}}} x_{2}, p^{\alpha_{1}} \overline{p^{\alpha_{2}}} y_{1}, \overline{p^{\alpha_{2}}} y_{2}, \overline{p^{\alpha_{1}}} z_{1}, \overline{p^{\alpha_{2}}} z_{2} ; t_{1}, t_{2}\right)
\end{aligned}
$$

whenever $\alpha_{1}, \alpha_{2} \geqslant 3$ and $p \nmid t_{1} t_{2}$, as well as

$$
\begin{aligned}
\widehat{S}_{a, b, d}\left(x_{1}, x_{2}, y_{1}, y_{2}, z_{1}, z_{2} ; t_{1} u_{1}, t_{2} u_{2}\right)= & \widehat{S}_{a, b, d}\left(\bar{t}_{1} x_{1}, t_{2} \bar{t}_{1} x_{2}, t_{1} \bar{t}_{2} y_{1}, \bar{t}_{2} y_{2}, \bar{t}_{1} z_{1}, \bar{t}_{2} z_{2} ; u_{1}, u_{2}\right) \\
& \times \widehat{S}_{a, b, d}\left(\bar{u}_{1} x_{1}, u_{2} \bar{u}_{1} x_{2}, u_{1} \bar{u}_{2} y_{1}, \bar{u}_{2} y_{2}, \bar{u}_{1} z_{1}, \bar{u}_{2} z_{2} ; t_{1}, t_{2}\right)
\end{aligned}
$$

whenever $\left(t_{1} t_{2}, u_{1} u_{2}\right)=1$. Here $a$ and $b$ on the right hand sides are understood as primitive residue classes in the respective smaller residue rings. 
Let $q$ be a prime. By (2.3) we have

$$
\begin{aligned}
& S^{(N)}\left(a m_{1} d, b n_{2} l_{2}, n_{1} l_{1}, m_{2} d ; q^{\alpha_{1}}, q^{\alpha_{2}}\right) \\
= & \sum_{\substack{B_{1}, C_{1}\left(\bmod q^{\alpha_{1}}\right) \\
B_{2}, C_{2}\left(\bmod q^{\alpha_{2}}\right)}} e\left(\frac{a m_{1} d B_{1}+n_{1} l_{1}\left(Y_{1} q^{\alpha_{2}}-Z_{1} B_{2}\right)}{q^{\alpha_{1}}}+\frac{b n_{2} l_{2} B_{2}+m_{2} d\left(Y_{2} q^{\alpha_{1}}-Z_{2} B_{1}\right)}{q^{\alpha_{2}}}\right)
\end{aligned}
$$

for $N \mid q^{\min \left(\alpha_{1}, \alpha_{2}\right)}$, where the sum is subject to

$$
q^{\alpha_{1}} C_{2}+B_{1} B_{2}+q^{\alpha_{2}} C_{1} \equiv 0\left(\bmod q^{\alpha_{1}+\alpha_{2}}\right), \quad\left(B_{j}, C_{j}, q\right)=1, \quad N \mid B_{1}
$$

and

$$
Y_{j} B_{j}+Z_{j} C_{j} \equiv 1\left(\bmod q^{\alpha_{j}}\right) \quad \text { for } \quad j=1,2 .
$$

We keep in mind that $q \nmid a b$. This sum is well-defined as shown in [BFG, Lemma 4.2], and does not depend on the choice of the representatives $B_{1}, B_{2}$. In particular, we can and will always assume

$$
1 \leqslant B_{j} \leqslant q^{\alpha_{j}} \text { for } j=1,2 \text {. }
$$

For future purposes we notice that (4.9) implies

$$
v_{q}\left(B_{1}\right) \leqslant \alpha_{2}, \quad v_{q}\left(B_{2}\right) \leqslant \alpha_{1}
$$

where $v_{q}$ denotes the $q$-adic valuation. Indeed, the first inequality is trivial if $\alpha_{1} \leqslant \alpha_{2}$. If $\alpha_{1}>\alpha_{2}$, the first condition in (4.9) implies $q^{\alpha_{2}} C_{1}+B_{1} B_{2} \equiv 0\left(\bmod q^{\alpha_{1}}\right)$. If $q \nmid B_{1}$, there is nothing to prove, otherwise we have $q \nmid C_{1}$, so $v_{q}\left(B_{1}\right) \leqslant v_{q}\left(B_{1} B_{2}\right)=\alpha_{2}$. Similarly one shows the second inequality.

Lemma 7. Let $q$ be a prime, and let $\alpha_{1}, \alpha_{2} \in \mathbb{N}_{0}$. For $x_{1}, y_{1}, z_{1}, x_{2}, y_{2}, z_{2} \in \mathbb{Z}$ and $d \in \mathbb{N}$ define

$$
\gamma:=\min \left(v_{q}\left(x_{1}\right), v_{q}\left(x_{2}\right), v_{q}\left(y_{1}\right), v_{q}\left(y_{2}\right), v_{q}\left(z_{1}\right), v_{q}\left(z_{2}\right)\right), \quad \delta=v_{q}(d) .
$$

Then we have

$$
\left|\widehat{S}_{a, b, d}\left(x_{1}, x_{2}, y_{1}, y_{2}, z_{1}, z_{2} ; q^{\alpha_{1}}, q^{\alpha_{2}}\right)\right| \leqslant 3 q^{2 \min \left(\alpha_{1}, \alpha_{2}\right)-\left(\alpha_{1}+\alpha_{2}\right)+2(\gamma+\delta)} .
$$

Here we apply the usual convention $\min (\infty, n)=n$ for $n \in \mathbb{N}_{0}$. The bound is meaningless for $x_{1}=x_{2}=$ $y_{1}=y_{2}=z_{1}=z_{2}=0$. This case will be considered in Lemma 9 . We defer the lengthy proof of Lemma 7 to the end of this section. A similar result holds for the twisted transform.

Lemma 8. Let $\chi$ be a primitive character modulo a prime $p$, and let $\alpha_{1}, \alpha_{2} \in \mathbb{N}_{0}$. For $x_{1}, x_{2}, y_{1}, y_{2}, z_{1}, z_{2} \in$ $\mathbb{Z}$ define

$$
\rho:=\min \left(v_{p}\left(x_{1}\right), v_{p}\left(x_{2}\right), v_{p}\left(y_{1}\right), v_{p}\left(y_{2}\right), v_{p}\left(z_{1}\right), v_{p}\left(z_{2}\right)\right) .
$$

Assume that $(d, p)=1$. Then we have

$$
\left|\widehat{S}_{a, b, d}^{\chi}\left(x_{1}, x_{2}, y_{1}, y_{2}, z_{1}, z_{2} ; p^{\alpha_{1}}, p^{\alpha_{2}}\right)\right| \leqslant 3 p^{2 \min \left(\alpha_{1}, \alpha_{2}\right)-\left(\alpha_{1}+\alpha_{2}\right)+2 \rho+5} .
$$

Proof. This follows from the previous lemma and the following simple observation. Let $\chi$ be a primitive character modulo $p$, and let $S$ be a $p^{\alpha}$-periodic function with $\alpha \geqslant 1$. Then

$$
\frac{1}{p^{\alpha}} \sum_{n\left(\bmod p^{\alpha}\right)} \chi(n) S(n) e\left(-\frac{n x}{p^{\alpha}}\right)=\frac{1}{\tau(\bar{\chi})} \sum_{\beta=1}^{p-1} \bar{\chi}(\beta) \frac{1}{p^{\alpha}} \sum_{n\left(\bmod p^{\alpha}\right)} S(n) e\left(-\frac{n\left(x+p^{\alpha-1} \beta\right)}{p^{\alpha}}\right)
$$

where as usual $\tau(\chi)$ denotes the Gauß sum (a complex number of absolute value $p^{1 / 2}$ ). We apply this formula for all six summation variables in (4.5) and estimate the various $\beta$-sums trivially (this produces an extra factor of $p^{6 / 2}=p^{3}$ ). Then we apply Lemma 7 with $\gamma \leqslant \rho+1$ and $\delta=0$.

Lemma 9. Let $\chi$ be a primitive non-quadratic character modulo a prime $p, \alpha_{1}, \alpha_{2} \geqslant 3,(d, p)=1$. Then

$$
\widehat{S}_{a, b, d}^{\chi}\left(0,0,0,0,0,0 ; p^{\alpha_{1}}, p^{\alpha_{2}}\right)=0 .
$$


Proof. We start with the observation that a Gauß sum

$$
\sum_{r\left(\bmod p^{\lambda}\right)} \chi(r) e\left(\frac{K r}{p^{\lambda}}\right)
$$

vanishes unless $v_{p}(K)=\lambda-1$.

The last condition in (4.9) implies that $p \mid B_{1}$, hence $p \nmid C_{1}$, and we can choose $Y_{1}=0, Z_{1}=\bar{C}_{1}$ in (4.10). Hence the $n_{1}, n_{2}$-sum becomes

$$
\sum_{n_{1}\left(\bmod p^{\alpha_{1}}\right)} \sum_{n_{2}\left(\bmod p^{\alpha_{2}}\right)} \bar{\chi}\left(n_{1}\right) \chi\left(n_{2}\right) e\left(-\frac{n_{1} l_{1} \bar{C}_{1} B_{2}}{p^{\alpha_{1}}}\right) e\left(\frac{b n_{2} l_{2} B_{2}}{p^{\alpha_{2}}}\right) .
$$

Since $\left(l_{1} l_{2}, p\right)=1$ by the presence of the character and $(b, p)=1$ by assumption, this is only non-zero if $\alpha_{1}=\alpha_{2}=\alpha \geqslant 3$, say, which we assume from now on. Moreover, $v_{p}\left(B_{2}\right)=\alpha-1$. Next, the sum over $m_{1}$ equals

$$
\sum_{m_{1}\left(\bmod p^{\alpha}\right)} \chi\left(m_{1}\right) e\left(\frac{a m_{1} d B_{1}}{p^{\alpha}}\right)
$$

which implies $v_{p}\left(B_{1}\right)=\alpha-1$. We write $B_{1}=p^{\alpha-1} \beta_{1}, B_{2}=p^{\alpha-1} \beta_{2}$ with $p \nmid \beta_{1}, \beta_{2}$. Then $C_{2} \equiv$ $-C_{1}-\beta_{1} \beta_{2} p^{\alpha-2}\left(\bmod p^{\alpha}\right)$, and the $B_{1}, B_{2}, C_{1}, C_{2}$-sum becomes

$$
\begin{aligned}
& \sum_{\substack{\beta_{1}, \beta_{2}(\bmod p) \\
\left(\beta_{1} \beta_{2}, p\right)=1}} \sum_{\substack{C_{1}\left(\bmod p^{\alpha}\right) \\
\left(C_{1}, p\right)=1}} e\left(\frac{a m_{1} d \beta_{1}-n_{1} l_{1} \bar{C}_{1} \beta_{2}}{p}+\frac{b n_{2} l_{2} \beta_{2}+m_{2} d \overline{\left(C_{1}+\beta_{1} \beta_{2} p^{\alpha-2}\right)} \beta_{1}}{p}\right) \\
= & p^{\alpha-1} \sum_{\substack{\beta_{1}, \beta_{2}, C_{1}(\bmod p) \\
\left(\beta_{1}, \beta_{2} C_{1}, p\right)=1}} e\left(\frac{a m_{1} d \beta_{1}-n_{1} l_{1} \bar{C}_{1} \beta_{2}}{p}+\frac{b n_{2} l_{2} \beta_{2}+m_{2} d \bar{C}_{1} \beta_{1}}{p}\right) .
\end{aligned}
$$

Here we use that $\alpha \geqslant 3$. The character implies that all variables in the numerator are coprime to $p$. Changing variables $\beta_{1} \mapsto \bar{m}_{1} \beta_{1}, \beta_{2} \mapsto \overline{n_{2} l_{2}} \beta_{2}, C_{1} \mapsto n_{1} l_{1} \overline{n_{2} l_{2}} C_{1}$, we see that this expression depends only on the product $\overline{m_{1} n_{1} l_{1}} m_{2} n_{2} l_{2}$, not on the six variables individually. Calling this expression $T\left(\overline{m_{1} n_{1} l_{1}} m_{2} n_{2} l_{2}\right)$, we obtain finally by another change of variables (e.g. $\left.m_{2} \mapsto m_{2} n_{1}\right)$ that

$$
\begin{aligned}
& \sum_{n_{1}, n_{2}, m_{1}, m_{2}, l_{1}, l_{2}\left(\bmod p^{\alpha}\right)} \bar{\chi}\left(n_{1} l_{1} m_{2}\right) \chi\left(n_{2} l_{2} m_{1}\right) T\left(\overline{m_{1} n_{1} l_{1}} m_{2} n_{2} l_{2}\right) \\
= & \sum_{n_{1}, n_{2}, m_{1}, m_{2}, l_{1}, l_{2}\left(\bmod p^{\alpha}\right)} \bar{\chi}\left(n_{1}^{2} l_{1} m_{2}\right) \chi\left(n_{2} l_{2} m_{1}\right) T\left(\overline{m_{1} l_{1}} m_{2} n_{2} l_{2}\right)
\end{aligned}
$$

and the $n_{1}$-sum vanishes since $\chi$ is not quadratic.

We combine the previous computations to the following useful result:

Corollary 10. Let $\chi$ be a primitive non-quadratic character modulo a prime $p$. Let $D_{1}, D_{2}, d \in \mathbb{N}$ satisfying $p^{3}\left|D_{1}, p^{3}\right| D_{2}$ and $(d, p)=1$. Let $x_{1}, x_{2}, y_{1}, y_{2}, z_{1}, z_{2} \in \mathbb{Z}$. Then

$$
\left|\widehat{S}_{a, b, d}^{\chi}\left(x_{1}, x_{2}, y_{1}, y_{2}, z_{1}, z_{2} ; D_{1}, D_{2}\right)\right| \leqslant p^{5} \tau_{3}\left(\left(D_{1}, D_{2}\right)\right) \frac{\left(D_{1}, D_{2}\right)^{2}}{D_{1} D_{2}}\left(x_{1}, x_{2}, y_{1}, y_{2}, z_{1}, z_{2}, D_{1}, D_{2}\right)^{2}\left(d, D_{1}, D_{2}\right)^{2}
$$

where $\tau_{3}$ is the ternary divisor function. Moreover,

$$
\widehat{S}_{a, b, d}^{\chi}\left(0,0,0,0,0,0 ; D_{1}, D_{2}\right)=0 .
$$

Indeed, the last statement is a direct consequence of (4.6) and Lemma 9. The first bound follows from the Chinese remainder theorem together with (4.6), (4.7) and Lemmas 7 and 8, noting that $\tau_{3}(q)=3$ for a prime $q$ and $q^{2 \min \left(\alpha_{1}, \alpha_{2}\right)-\left(\alpha_{1}+\alpha_{2}\right)}=\left(q_{1}^{\alpha_{1}}, q_{2}^{\alpha_{2}}\right)^{2} /\left(q_{1}^{\alpha_{1}} q_{2}^{\alpha_{2}}\right)$. 
Finally we give the proof of Lemma 7 . We will frequently use the following simple result

$$
\left|\sum_{n, l\left(\bmod q^{\alpha}\right)} e\left(\frac{n l B}{q^{\alpha}}\right) e\left(-\frac{n x-l z}{q^{\alpha}}\right)\right|= \begin{cases}q^{\alpha+v_{q}(B)}, & v_{q}(B) \leqslant \min \left(v_{q}(x), v_{q}(z)\right) \\ 0, & \text { otherwise }\end{cases}
$$

for integers $x, z$ and $v_{q}(B) \leqslant \alpha$. Indeed, the $n$-sum vanishes unless $l B-x \equiv 0\left(\bmod q^{\alpha}\right)$ in which case it equals $q^{\alpha}$. This implies in particular $v_{q}(B) \leqslant v_{q}(x)$ and defines $l$ modulo $q^{\alpha-v_{q}(B)}$. Then the $l$-sum vanishes unless $v_{q}(z) \geqslant v_{q}(B)$, and the result follows easily.

We will now distinguish several cases to estimate $\widehat{S}_{a, b, d}$, but the overall strategy is always the same. We open the Kloosterman sum and pull the $B_{1}, B_{2}, C_{1}, C_{2}$-sums outside. Then we sum over $n_{1}, n_{2}, l_{1}, l_{2}, m_{1}, m_{2}$ using orthogonality of additive characters or (4.13). At this point we estimate trivially and just count how many quadruples $\left(B_{1}, B_{2}, C_{1}, C_{2}\right)$ survive in the outer sum.

In order to avoid pathological cases, we treat the case $\alpha_{1} \alpha_{2}=0$ separately. The case $\alpha_{1}=\alpha_{2}=0$ is trivial, so let us assume $\alpha_{1}>0, \alpha_{2}=0$. In this case the $w_{6}$-Kloosterman sum degenerates to an ordinary Kloosterman sum

$$
S^{(1)}\left(a m_{1} d, b n_{2} l_{2}, n_{1} l_{1}, m_{2} d, q^{\alpha_{1}}, 1\right)=S\left(a m_{1} d, n_{1} l_{1}, q^{\alpha_{1}}\right)
$$

by [BFG, Property 4.9], so that we need to bound

$$
\frac{1}{q^{3 \alpha_{1}}} \sum_{n_{1}, l_{1}, m_{1}\left(\bmod q^{\alpha_{1}}\right)} S\left(a m_{1} d, n_{1} l_{1}, q^{\alpha_{1}}\right) e\left(\frac{-n_{1} x_{1}-m_{1} y_{1}-l_{1} z_{1}}{q^{\alpha_{1}}}\right) .
$$

We open the Kloosterman sum, sum over $n_{1}, l_{1}$ by (4.13) and over $m_{1}$ by orthogonality of characters getting the bound $q^{-\alpha_{1}+\delta}$. The case $\alpha_{1}=0, \alpha_{2}>0$ is similar. From now on we assume $\alpha_{1}>0$ and $\alpha_{2}>0$.

We return to the definition (4.4) and split the Kloosterman sum in (4.8) into two parts according to whether $q \nmid C_{1}$ or $q \mid C_{1}$. This gives a decomposition $\widehat{S}_{a, b, d}=T_{a, b, d}+U_{a, b, d}$. The second term where $q \mid C_{1}$ is easier. In this case $q \nmid B_{1}$ by (4.9), and the first condition there implies $q \mid B_{2}$ (since $\alpha_{1}, \alpha_{2}>0$ ), so that $q \nmid C_{2}$. Hence we can choose

$$
Y_{1}=\bar{B}_{1}, \quad Z_{2}=\bar{C}_{2}, \quad Z_{1}=Y_{2}=0 .
$$

Considering the $q$-powers in the first condition in (4.9) again, we see that $v_{q}\left(B_{1} B_{2}\right) \leqslant \alpha_{2}$, but $v_{q}\left(q^{\alpha_{2}} C_{1}+\right.$ $\left.q^{\alpha_{1}} C_{2}\right) \geqslant \min \left(\alpha_{1}, \alpha_{2}+1\right)$, and we conclude $\alpha_{1} \leqslant \alpha_{2}$. Thus

$$
\begin{aligned}
& U_{a, b, d}=\frac{1}{q^{3\left(\alpha_{1}+\alpha_{2}\right)}} \sum_{\substack{B_{1}, C_{1}\left(\bmod q^{\alpha_{1}}\right) \\
B_{2}, C_{2}\left(\bmod q^{\alpha_{2}}\right)}} \sum_{\substack{n_{1}, m_{1}, l_{1}\left(\bmod q^{\alpha_{1}}\right) \\
n_{2}, m_{2}, l_{2}\left(\bmod q^{\alpha_{2}}\right)}} e\left(\frac{a m_{1} d B_{1}}{q^{\alpha_{1}}}+\frac{b n_{2} l_{2} B_{2}-m_{2} d \bar{C}_{2} B_{1}}{q^{\alpha_{2}}}\right) \\
& \text { (4.9) holds with } N=1 \\
& q \nmid B_{1} C_{2}, q\left|C_{1}, q\right| B_{2} \\
& \times e\left(-\frac{n_{1} x_{1}+m_{1} y_{1}+l_{1} z_{1}}{q^{\alpha_{1}}}\right) e\left(-\frac{n_{2} x_{2}+m_{2} y_{2}+l_{2} z_{2}}{q^{\alpha_{2}}}\right) .
\end{aligned}
$$

We sum trivially over $n_{1}, l_{1}$, we use (4.13) in combination with (4.11) for the sum over $n_{2}, l_{2}$, and we sum over $m_{1}, m_{2}$ using orthogonality of characters. The latter two sums leave $q^{\delta}$ choices for $B_{1}$ and $C_{2}$ respectively (recall that $q \nmid B_{1}$ ). Now the first condition in (4.9) determines $B_{2}$ modulo $q^{\alpha_{2}}$ which then determines $C_{1}$. Altogether we obtain

$$
\left|U_{a, b, d}\right| \leqslant q^{\alpha_{1}-\alpha_{2}+2 \delta}=q^{2 \min \left(\alpha_{1}, \alpha_{2}\right)-\alpha_{1}-\alpha_{2}+2 \delta} .
$$

Now we turn to the estimation of $T_{a, b, d}$ where $q \nmid C_{1}$, so that

$$
Z_{1}=\bar{C}_{1}, \quad Y_{1}=0
$$


and we obtain

$$
\begin{aligned}
T_{a, b, d}= & \frac{1}{q^{3\left(\alpha_{1}+\alpha_{2}\right)}} \sum_{\begin{array}{c}
B_{1}, C_{1}\left(\bmod q^{\alpha_{1}}\right) \\
B_{2}, C_{2}\left(\bmod q^{\alpha_{2}}\right) \\
(4.9) \text { holds with } N=1 \\
q \nmid C_{1}
\end{array}} \sum_{\substack{n_{1}, m_{1}, l_{1}\left(\bmod q_{2} q_{1}, l_{2}\left(\bmod q^{\alpha_{2}}\right) \\
n_{2}, m_{2}\right.}} e\left(-\frac{n_{1} x_{1}+m_{1} y_{1}+l_{1} z_{1}}{q^{\alpha_{1}}}\right) \\
& \times e\left(-\frac{n_{2} x_{2}+m_{2} y_{2}+l_{2} z_{2}}{q^{\alpha_{2}}}\right) e\left(\frac{a m_{1} d B_{1}-n_{1} l_{1} \bar{C}_{1} B_{2}}{q^{\alpha_{1}}}+\frac{b n_{2} l_{2} B_{2}+m_{2} d\left(Y_{2} q^{\alpha_{1}}-Z_{2} B_{1}\right)}{q^{\alpha_{2}}}\right) .
\end{aligned}
$$

By (4.13), the sum over $n_{2}, l_{2}$ contributes $q^{\alpha_{2}+v_{q}\left(B_{2}\right)}$ if $v_{q}\left(B_{2}\right) \leqslant \min \left(v_{q}\left(x_{2}\right), v_{q}\left(z_{2}\right)\right)$ and is zero otherwise. Similarly the sum over $n_{1}, l_{1}$ contributes $q^{\alpha_{1}+v_{q}\left(B_{2}\right)}$ if $v_{q}\left(B_{2}\right) \leqslant \min \left(v_{q}\left(x_{1}\right), v_{q}\left(z_{1}\right)\right)$ and is zero otherwise (note that by (4.11) we have $v_{q}\left(B_{2}\right) \leqslant \alpha_{1}$, so that (4.13) is applicable). We conclude that the combined sum over $n_{1}, n_{2}, l_{1}, l_{2}$ contributes

$$
q^{\alpha_{1}+\alpha_{2}+2 \min \left(v_{q}\left(x_{1}\right), v_{q}\left(x_{2}\right), v_{q}\left(z_{1}\right), v_{q}\left(z_{2}\right)\right)} .
$$

As before the $m_{1}$-sum leaves at most $q^{\delta}$ choices for $B_{1}$, and we fix one of them.

Let us first assume that $q \nmid B_{2}$, so that

$$
Y_{2}=\bar{B}_{2}, \quad Z_{2}=0
$$

Then the $m_{2}$-sum leaves at most $q^{\alpha_{1}+\delta}$ choices for $B_{2}$ (and trivially there are at most $q^{\alpha_{2}}$ ) choices for $\left.B_{2}\right)$. Again we fix one of them. If $\alpha_{1} \leqslant \alpha_{2}$ fix a choice for $C_{1}$, otherwise fix a choice for $C_{2}$. In either case, the other $C$-variable is determined by (4.9). We conclude that there are in total at most $q^{\delta+\min \left(\alpha_{1}+\delta, \alpha_{2}\right)+\min \left(\alpha_{1}, \alpha_{2}\right)} \leqslant q^{2 \min \left(\alpha_{1}, \alpha_{2}\right)+2 \delta}$ choices for the quadruples $\left(B_{1}, B_{2}, C_{1}, C_{2}\right)$ satisfying $q \nmid B_{2}$.

Let us now assume $q \mid B_{2}$, so that $q \nmid C_{2}$ and

$$
Y_{2}=0, \quad Z_{2}=\bar{C}_{2}
$$

The $m_{2}$-sum leaves at most $q^{\delta+v_{q}\left(B_{1}\right)} \leqslant q^{\delta+\alpha_{1}}$ choices for $C_{2}$ (and trivially there are at most $q^{\alpha_{2}}$ choices for $C_{2}$ ). If $\alpha_{1} \leqslant \alpha_{2}$ fix a choice for $C_{1}$, otherwise fix a choice for $B_{2}$. In either case, the other variable is determined by (4.9). As above we conclude that there are in total at most $q^{2 \min \left(\alpha_{1}, \alpha_{2}\right)+2 \delta}$ choices for the quadruples $\left(B_{1}, B_{2}, C_{1}, C_{2}\right)$ satisfying $q \mid B_{2}$.

We conclude from the previous discussion that the sum over $m_{1}, m_{2}$ together with the sum over $B_{1}, B_{2}, C_{1}, C_{2}$ contributes $2 q^{\alpha_{1}+\alpha_{2}+2 \min \left(\alpha_{1}, \alpha_{2}\right)+2 \delta}$, and we obtain by (4.16) the total bound

$$
\left|T_{a, b, d}\right| \leqslant 2 q^{2 \min \left(\alpha_{1}, \alpha_{2}\right)-\alpha_{1}-\alpha_{2}+2 \min \left(v_{q}\left(x_{1}\right), v_{q}\left(x_{2}\right), v_{q}\left(z_{1}\right), v_{q}\left(z_{2}\right)\right)+2 \delta} .
$$

This is not quite sufficient to substantiate the claim of Lemma 7, so we proceed to prove an alternative bound for $T_{a, b, d}$ as defined in (4.15). Let us first assume that $q \nmid B_{2}$, so that (4.17) holds. Then by (4.13), the $n_{1}, l_{1}, n_{2}, l_{2}$-sums contribute $q^{\alpha_{1}+\alpha_{2}}$, while the $m_{1}, m_{2}$-sums leave as above $q^{\delta+\min \left(\alpha_{1}+\delta, \alpha_{2}\right)}$ choices for the pair $\left(B_{1}, B_{2}\right)$. Fix a choice for $C_{1}$ if $\alpha_{1} \leqslant \alpha_{2}$, otherwise fix a choice for $C_{2}$; in either case the other variable is determined by (4.9). In total we obtain at most $q^{2 \delta+2 \min \left(\alpha_{1}, \alpha_{2}\right)}$ choices for the quadruples $\left(B_{1}, B_{2}, C_{1}, C_{2}\right)$, so that together with the $m_{1}, m_{2}$-sum we obtain a total contribution of

$$
q^{2 \min \left(\alpha_{1}, \alpha_{2}\right)-\alpha_{1}-\alpha_{2}+2 \delta}
$$

for the terms $q \nmid B_{2}$.

Let us now consider the terms with $q \mid B_{2}$, so that $q \nmid C_{2}$ and

$$
Y_{2}=0, \quad Z_{2}=\bar{C}_{2},
$$


so that (4.15) simplifies to

$$
\begin{aligned}
& \frac{1}{q^{3\left(\alpha_{1}+\alpha_{2}\right)}} \sum_{B_{1}, C_{1}\left(\bmod q^{\alpha_{1}}\right)} \sum_{n_{1}, m_{1}, l_{1}\left(\bmod q^{\alpha_{1}}\right)} e\left(\frac{a m_{1} d B_{1}-n_{1} l_{1} \bar{C}_{1} B_{2}}{q^{\alpha_{1}}}+\frac{b n_{2} l_{2} B_{2}-m_{2} d \bar{C}_{2} B_{1}}{q^{\alpha_{2}}}\right) \\
& B_{2}, C_{2}\left(\bmod q^{\alpha_{2}}\right) \quad n_{2}, m_{2}, l_{2}\left(\bmod q^{\alpha_{2}}\right) \\
& \text { (4.9) holds with } N=1 \\
& q \nmid C_{1} C_{2}, q \mid B_{2} \\
& \times e\left(-\frac{n_{1} x_{1}+m_{1} y_{1}+l_{1} z_{1}}{q^{\alpha_{1}}}\right) e\left(-\frac{n_{2} x_{2}+m_{2} y_{2}+l_{2} z_{2}}{q^{\alpha_{2}}}\right) .
\end{aligned}
$$

In the following we assume without loss of generality $1 \leqslant y_{1} \leqslant q^{\alpha_{1}}$ and $1 \leqslant y_{2} \leqslant q^{\alpha_{2}}$, so that $v_{q}\left(y_{1}\right) \leqslant \alpha_{1}$ and $v_{q}\left(y_{2}\right) \leqslant \alpha_{2}$. It is convenient to first dispense with the case $\min \left(v_{q}\left(y_{1}\right), v_{q}\left(y_{2}\right)\right) \geqslant \alpha_{2}\left(\right.$ and hence $\left.=\alpha_{2}\right)$. Here the $n_{1}, n_{2}, l_{1}, l_{2}$-sums contribute by $(4.13)$ and (4.11) at most $q^{\alpha_{1}+\alpha_{2}+2 \min \left(\alpha_{1}, \alpha_{2}\right)}$ while there are at most $q^{\delta}$ choices for $B_{1}$ and trivially at most $q^{2 \alpha_{2}} \leqslant q^{2 \min \left(v_{q}\left(y_{1}\right), v_{q}\left(y_{2}\right)\right)}$ choices for $\left(B_{2}, C_{2}\right)$ which determines $C_{1}$. This gives the total bound

$$
q^{2 \min \left(\alpha_{1}, \alpha_{2}\right)-\alpha_{1}-\alpha_{2}+2 \min \left(v_{q}\left(y_{1}\right), v_{q}\left(y_{2}\right)\right)+\delta}
$$

for (4.20) under the present assumption $\min \left(v_{q}\left(y_{1}\right), v_{q}\left(y_{2}\right)\right) \geqslant \alpha_{2}$. From now on we assume

$$
\min \left(v_{q}\left(y_{1}\right), v_{q}\left(y_{2}\right)\right)<\alpha_{2} \text {. }
$$

We distinguish two cases.

Let us first assume $v_{q}\left(y_{1}\right) \leqslant v_{q}\left(y_{2}\right)$. By (4.13) and (4.11) the sum over $n_{1}, l_{1}, n_{2}, l_{2}$ contributes at most $q^{\alpha_{1}+\alpha_{2}+2 \min \left(\alpha_{1}, \alpha_{2}\right)}$. The $m_{1}$-sum leaves at most $q^{\delta}$ choices for $B_{1}$ and each of them satisfies $v_{q}\left(B_{1}\right) \leqslant v_{q}\left(y_{1}\right)$. We fix one of them. Similarly then the sum over $m_{2}$ leaves at most $q^{\delta+v_{q}\left(B_{1}\right)} \leqslant q^{\delta+v_{q}\left(y_{1}\right)}$ choices for $C_{2}$. If $B_{1}, C_{2}$ are fixed, then the first condition in (4.9) leaves at most $q^{v_{q}\left(B_{1}\right)} \leqslant q^{v_{q}\left(y_{1}\right)}$ choices for $B_{2}$ modulo $q^{\alpha_{2}}$, and the triple $B_{1}, B_{2}, C_{2}$ determines $C_{1}$. We conclude that there are at most $q^{2 v_{q}\left(y_{1}\right)+2 \delta}$ choices for the quadruple $\left(B_{1}, B_{2}, C_{1}, C_{2}\right)$, and we obtain the total bound

$$
q^{2 \min \left(\alpha_{1}, \alpha_{2}\right)-\alpha_{1}-\alpha_{2}+2 \min \left(v_{q}\left(y_{1}\right), v_{q}\left(y_{2}\right)\right)+2 \delta} .
$$

for (4.20) under the present assumption (4.22) and $v_{q}\left(y_{1}\right) \leqslant v_{q}\left(y_{2}\right)$. This bound dominates (4.21).

The other case $v_{q}\left(y_{1}\right)>v_{q}\left(y_{2}\right)$ cannot happen: first we observe that the $m_{1}, m_{2}$-sum vanishes unless $d B_{1} \equiv \bar{a} y_{1}\left(\bmod q^{\alpha_{1}}\right)$ and $d B_{1} \equiv-C_{2} y_{2}\left(\bmod q^{\alpha_{2}}\right)$. Together with (4.22) this leads to a contradiction unless $v_{q}\left(y_{2}\right) \geqslant \alpha_{1}$. But this is impossible since $\alpha_{1} \geqslant v_{q}\left(y_{1}\right)>v_{q}\left(y_{2}\right)$. We summarize that (4.23) is an upper bound for (4.20) in all cases, and together with (4.19) we conclude

$$
\left|T_{a, b, d}\right| \leqslant 2 q^{2 \min \left(\alpha_{1}, \alpha_{2}\right)-\alpha_{1}-\alpha_{2}+2 \min \left(v_{q}\left(y_{1}\right), v_{q}\left(y_{2}\right)\right)+2 \delta} .
$$

Combining (4.18) and (4.24) with (4.14) completes the proof of Lemma 7.

\section{The SiXth MOMEnT}

5.1. Setting up the Kuznetsov formula. We prepare now for the proof of Theorem 1 . We recall the setup that $N$ is a large prime, $p$ is a fixed prime and $\chi$ is a primitive non-quadratic character modulo $p$. All implied constants may depend on $p$. Let $\pi \subseteq L_{\text {cusp }}^{2}\left(\Gamma_{0}(N) \backslash \mathbb{H}_{3}\right)$ be a cuspidal automorphic representation. Its $L$-function has conductor dividing $N$ [JPSS, Théorème]. The contribution in the moment estimate of Theorem 1 of those $\pi$ with conductor 1 is independent of $N$, hence $O(1)$, therefore it suffices to consider $\pi$ of conductor $N$. Fix a newvector $\varpi \in \pi$ and denote its normalized Fourier coefficients, defined in (2.7) and (2.8), with $A_{\varpi}(1,1)=1$. By an approximate functional equation [IK, Theorem 5.2] we have

$$
|L(1 / 2, \pi \times \chi)|^{2}=\left|\sum_{n} \frac{a_{\pi \times \chi}(n)}{n^{1 / 2}} V\left(\frac{n}{N^{1 / 2}}\right)+\eta \sum_{n} \frac{\overline{a_{\pi \times \chi}(n)}}{n^{1 / 2}} V\left(\frac{n}{N^{1 / 2}}\right)\right|^{2}
$$

where $a_{\pi \times \chi}(n)$ are the Dirichlet coefficients of $L(s, \pi \times \chi), V$ is a smooth, bounded, rapidly decaying function depending on $\pi$ and $p$, and $\eta$ is a complex number of absolute value 1 depending on $\pi$ and 
$p$. The coefficients $a_{\pi \times \chi}(n)$ are multiplicative and satisfy $a_{\pi \times \chi}(n)=A_{\varpi}(n, 1) \chi(n)$ for $(n, N p)=1$, and $a_{\pi \times \chi}(n) \ll n^{5 / 14+\varepsilon}$ by known bounds towards the Ramanujan conjecture on GL(3) (although much weaker bounds would suffice for our purpose). Thus we have

$$
L(s, \pi \times \chi)=\sum_{n} \frac{A_{\varpi}(n, 1) \chi(n)}{n^{s}} \sum_{\nu=0}^{\infty} \frac{a_{\pi \times \chi}\left(p^{\nu}\right)}{p^{\nu s}} L_{N}(s)
$$

for a certain Euler factor $L_{N}(s)$.

We truncate the sums at $n \leqslant N^{1 / 2+\varepsilon}$ at the cost of a negligible error. Writing $V$ as its inverse Mellin transform, moving the contour to real part $\varepsilon$ and pulling the rapidly converging integral outside the absolute values, we obtain

$$
|L(1 / 2, \pi \times \chi)|^{2} \ll N^{\varepsilon} \int_{|t| \leqslant N^{\varepsilon}}\left|\sum_{n \leqslant N^{1 / 2+\varepsilon}} \frac{a_{\pi \times \chi}(n)}{n^{1 / 2+\varepsilon+i t}}\right|^{2} d t .
$$

Coupled with a smooth partition of unity and the Cauchy-Schwarz inequality we have for some compactly supported weight functions $W_{j}$ (independent of $\pi$ ) that

$$
|L(1 / 2, \pi \times \chi)|^{2} \ll N^{\varepsilon} \int_{|t| \leqslant N^{\varepsilon}}\left|\sum_{2^{j} \leqslant N^{1 / 2+\varepsilon}} \sum_{n} \frac{a_{\pi \times \chi}(n)}{n^{1 / 2+i t}} W_{j}\left(\frac{n}{2^{j}}\right)\right|^{2} d t,
$$

up to a negligible error. Since $N$ is prime, the coefficients of the Euler factor $L_{N}(s)$ are irrelevant (for $\varepsilon<1 / 2)$. Estimating the coefficients $a_{\pi \times \chi}\left(p^{\nu}\right)$ trivially, we conclude

$$
\begin{aligned}
& |L(1 / 2, \pi \times \chi)|^{2} \ll N^{\varepsilon} \sum_{\nu} \frac{1}{p^{\nu\left(\frac{1}{2}-\frac{5}{14}-\varepsilon\right)}} \int_{|t| \leqslant N^{\varepsilon}}\left|\sum_{2^{j} \leqslant N^{1 / 2+\varepsilon}} \sum_{n} \frac{A_{\varpi}(n, 1) \chi(n)}{n^{1 / 2+i t}} W_{j}\left(\frac{n p^{\nu}}{2^{j}}\right)\right|^{2} d t \\
& \ll N^{\varepsilon} \sum_{\nu} \frac{1}{p^{\nu / 8}} \int_{|t| \leqslant N^{\varepsilon}} \sum_{2^{j} \leqslant N^{1 / 2+\varepsilon}} \sum_{n_{1}, n_{2}} \frac{A_{\varpi}\left(n_{1}, 1\right) \overline{A_{\varpi}\left(n_{2}, 1\right)} \chi\left(n_{1}\right) \bar{\chi}\left(n_{2}\right)}{\left(n_{1} n_{2}\right)^{1 / 2}}\left(\frac{n_{2}}{n_{1}}\right)^{i t} W_{j}\left(\frac{n_{1} p^{\nu}}{2^{j}}\right) \overline{W_{j}\left(\frac{n_{2} p^{\nu}}{2^{j}}\right)} d t .
\end{aligned}
$$

We observe that the $n_{1}, n_{2}$-sum is non-negative and that (for $\varepsilon<1 / 2$ ) the variables $n_{1}, n_{2}$ are coprime to $N$, so that the Fourier coefficients satisfy the unramified Hecke relations, as discussed prior to the statement of Theorem 6 (recall that for $\varpi$ with $A_{\varpi}(1,1)=0$ all coefficients coming up in the previous sum vanish). We multiply three such expressions together. Applying Hölder's inequality to the combined $\nu$-sum and $t$-integral with exponents $2 / 3$ and $1 / 3$ and using that

$$
\left(\sum_{\nu} \int_{|t| \leqslant N^{\varepsilon}}\left(\frac{1}{p^{\nu / 8}}\right)^{3 / 2}\right)^{2 / 3} \ll N^{\varepsilon}
$$

we obtain

$$
\begin{aligned}
|L(1 / 2, \pi \times \chi)|^{6} \ll N^{\varepsilon} & \sum_{\nu} \frac{1}{p^{\nu / 8}} \int_{|t| \leqslant N^{\varepsilon}} \sum_{2^{j \leqslant N^{1 / 2+\varepsilon}}} \sum_{\substack{n_{1}, m_{1}, l_{1} \\
n_{2}, m_{2}, l_{2}}} \chi\left(n_{1} m_{1} l_{1}\right) \bar{\chi}\left(n_{2} m_{2} l_{2}\right)\left(\frac{n_{2} m_{2} l_{2}}{n_{1} m_{1} l_{1}}\right)^{i t} \\
& \times \frac{A_{\varpi}\left(n_{1}, 1\right) \overline{A_{\varpi}\left(n_{2}, 1\right)} A_{\varpi}\left(m_{1}, 1\right) \overline{A_{\varpi}\left(m_{2}, 1\right)} A_{\varpi}\left(l_{1}, 1\right) \overline{A_{\varpi}\left(l_{2}, 1\right)}}{\left(n_{1} n_{2} m_{1} m_{2} l_{1} l_{2}\right)^{1 / 2}} \\
& \times W_{j}\left(\frac{n_{1} p^{\nu}}{2^{j}}\right) \overline{W_{j}\left(\frac{n_{2} p^{\nu}}{2^{j}}\right)} W_{j}\left(\frac{m_{1} p^{\nu}}{2^{j}}\right) \overline{W_{j}\left(\frac{m_{2} p^{\nu}}{2^{j}}\right)} W_{j}\left(\frac{l_{1} p^{\nu}}{2^{j}}\right) \overline{W_{j}\left(\frac{l_{2} p^{\nu}}{2^{j}}\right)} d t .
\end{aligned}
$$


Finally we multiply this with $\sum_{i}\left|\left\langle F_{i}, \tilde{W}_{\mu_{\pi}}\right\rangle\right|^{2}$ where $F_{i}$ is a collection of functions as in Lemma 1 and sum over $\pi$. This gives

$$
\begin{aligned}
& \sum_{\substack{\pi \subseteq L_{\text {cusp }}^{2}\left(\Gamma_{0}(N) \backslash \mathbb{H}_{3}\right) \\
\mu_{\pi} \in \Omega}}|L(1 / 2, \pi \times \chi)|^{6} \ll N^{\varepsilon} \max _{i} \max _{|t| \leqslant N^{\varepsilon}} \max _{M \leqslant N^{1 / 2+\varepsilon}} \sum_{\pi \subseteq L_{\text {cusp }}^{2}\left(\Gamma_{0}(N) \backslash \mathbb{H}_{3}\right)}\left|\left\langle F_{i}, \tilde{W}_{\mu_{\pi}}\right\rangle\right|^{2} \\
& \times\left|\sum_{n_{1}, m_{2}, l_{1}} \frac{A_{\varpi}\left(n_{1}, 1\right) \overline{A_{\varpi}\left(m_{2}, 1\right)} A_{\varpi}\left(l_{1}, 1\right) \chi\left(n_{1} l_{1}\right) \bar{\chi}\left(m_{2}\right)}{\left(n_{1} m_{2} l_{1}\right)^{1 / 2}} W\left(\frac{n_{1}}{M}\right) \overline{W\left(\frac{m_{2}}{M}\right)} W\left(\frac{l_{1}}{M}\right)\right|^{2}
\end{aligned}
$$

for some smooth compactly supported weight function $W$. In the interest of readable and compact notation let us introduce

$$
\sum_{m \sim M} f(m):=\sum_{m} f(m) W\left(\frac{m}{M}\right)
$$

for some unspecified smooth compactly supported weight function satisfying

$$
W^{(j)} \ll_{\varepsilon, j} N^{\varepsilon}
$$

for all $j \in \mathbb{N}_{0}$. In other words, $\sim$ has the same meaning as $\asymp$ except that an additional smooth weight function is attached to the sum which comes in handy when one applies Poisson summation.

We now use the Hecke relation [Go, Theorem 6.4.11]

$$
A_{\varpi}(n, 1) \overline{A_{\varpi}(m, 1)} A_{\varpi}(l, 1)=\sum_{\substack{d_{1} d_{2}\left|n \\ d_{1} d_{3}\right| m \\ d_{2} d_{3} \mid l}} A_{\varpi}\left(\frac{n l}{d_{1} d_{2}^{2} d_{3}}, \frac{m d_{2}}{d_{1} d_{3}}\right) .
$$

By (2.9) and another application of the Cauchy-Schwarz inequality applied to the $d_{1}, d_{2}, d_{3}$-sum, we obtain

$$
\begin{aligned}
\sum_{\substack{\pi \subseteq L_{\text {cusp }}^{2}\left(\Gamma_{0}(N) \backslash \mathbb{H}_{3}\right) \\
\mu_{\pi} \in \Omega}}|L(1 / 2, \pi \times \chi)|^{6} & \ll \max _{i} \max _{M \leqslant N^{1 / 2+\varepsilon}} \frac{N^{2+\varepsilon}}{M^{3}} \sum_{\pi \subseteq L_{\text {cusp }}^{2}\left(\Gamma_{0}(N) \backslash \mathbb{H}_{3}\right)} \frac{\left|\left\langle F_{i}, \tilde{W}_{\mu_{\pi}}\right\rangle\right|^{2}}{\mathcal{N}(\varpi)} \\
& \times \sum_{\substack{d_{1}, d_{2}, d_{3} \\
\left(d_{1} d_{2} d_{3}, p\right)=1}}\left|\sum_{\substack{n \sim M / d_{1} d_{2} \\
m \sim M / d_{1} d_{3} \\
l \sim M / d_{2} d_{3}}} A_{\varpi}\left(n l, m d_{2}\right) \chi(n l) \bar{\chi}(m)\right|^{2} .
\end{aligned}
$$

By positivity, we can add the rest of the spectrum. For technical reasons it convenient to sum over the spectrum of $L^{2}\left(\Gamma_{0}\left(p^{3} N\right) \backslash \mathbb{H}_{3}\right)$ which contains the sum in the preceding display as oldforms. We open the square and exchange summations. This gives finally our basic inequality

$$
\begin{aligned}
\sum_{\substack{\pi \subseteq L_{\text {cusp }}^{2}\left(\Gamma_{0}(N) \backslash \mathbb{H}_{3}\right) \\
\mu_{\pi} \in \Omega}}|L(1 / 2, \pi \times \chi)|^{6} & \ll \max _{i} \max _{M \leqslant N^{1 / 2+\varepsilon}} \frac{N^{2+\varepsilon}}{M^{3}} \sum_{\substack{d_{1}, d_{2}, d_{3} \\
\left(d_{1} d_{2} d_{3}, p\right)=1}} \sum_{\substack{n_{1}, n_{2} \sim M / d_{1} d_{2} \\
m_{1}, m_{2} \sim M / d_{1} d_{3} \\
l_{1}, l_{2} \sim M / d_{2} d_{3}}} \bar{\chi}\left(n_{1} l_{1} m_{2}\right) \chi\left(m_{1} n_{2} l_{2}\right) \\
& \times \int_{\left(p^{3} N\right)} \overline{A_{\varpi}\left(n_{1} l_{1}, m_{2} d_{2}\right)} A_{\varpi}\left(n_{2} l_{2}, m_{1} d_{2}\right) \frac{\left|\left\langle F_{i}, \tilde{W}_{\mu_{\pi}}\right\rangle\right|^{2}}{\mathcal{N}(\varpi)} d \varpi .
\end{aligned}
$$

5.2. Bounding the Kloosterman terms. The spectral term is now in shape for an application of the Kuznetsov formula (Theorem 6), and accordingly we write the right hand side as a sum of four terms 
$\Delta+\Sigma_{4}+\Sigma_{5}+\Sigma_{6}$, where

$$
\begin{aligned}
& \Delta=\max _{i} \max _{M \leqslant N^{1 / 2+\varepsilon}} \frac{N^{2+\varepsilon}}{M^{3}} \sum_{\substack{d_{1}, d_{2}, d_{3} \\
\left(d_{1} d_{2} d_{3}, p\right)=1}} \sum_{\begin{array}{c}
n_{1}, n_{2} \sim M / d_{1} d_{2} \\
m_{1}, m_{2} \sim M / d_{1} d_{3} \\
l_{1}, l_{2} \sim M / d_{2} d_{3}
\end{array}} \bar{\chi}\left(n_{1} l_{1} m_{2}\right) \chi\left(m_{1} n_{2} l_{2}\right) \delta_{\substack{n_{1} l_{1}=n_{2} l_{2} \\
m_{2}=m_{1}}}\left\|F_{i}\right\|^{2}, \\
& \Sigma_{4}=\max _{i} \max _{M \leqslant N^{1 / 2+\varepsilon}} \frac{N^{2+\varepsilon}}{M^{3}} \sum_{\substack{d_{1}, d_{2}, d_{3} \\
\left(d_{1} d_{2} d_{3}, p\right)=1}} \sum_{\begin{array}{c}
n_{1}, n_{2} \sim M / d_{1} d_{2} \\
m_{1}, m_{2} \sim M / d_{1} d_{3} \\
l_{1}, l_{2} \sim M / d_{2} d_{3}
\end{array}} \bar{\chi}\left(n_{1} l_{1} m_{2}\right) \chi\left(m_{1} n_{2} l_{2}\right) \\
& \sum_{\epsilon= \pm 1} \sum_{\substack{p^{3} N D_{2} \mid D_{1} \\
d_{2} m_{2} D_{1}=l_{2} n_{2} D_{2}^{2}}} \frac{\tilde{S}\left(\epsilon d_{2} m_{1}, d_{2} m_{2}, l_{1} n_{1}, D_{2}, D_{1}\right)}{D_{1} D_{2}} \tilde{\mathcal{J}}_{\epsilon ; F_{i}^{*}}\left(\sqrt{\frac{l_{1} n_{1} d_{2}^{2} m_{1} m_{2}}{D_{1} D_{2}}}\right) \\
& \Sigma_{5}=\max _{i} \max _{M \leqslant N^{1 / 2+\varepsilon}} \frac{N^{2+\varepsilon}}{M^{3}} \sum_{\begin{array}{c}
d_{1}, d_{2}, d_{3} \\
\left(d_{1} d_{2} d_{3}, p\right)=1
\end{array}} \sum_{\begin{array}{c}
n_{1}, n_{2} \sim M / d_{1} d_{2} \\
m_{1}, m_{2} \sim M / d_{1} d_{3} \\
l_{1}, l_{2} \sim M / d_{2} d_{3}
\end{array}} \bar{\chi}\left(n_{1} l_{1} m_{2}\right) \chi\left(m_{1} n_{2} l_{2}\right) \\
& \sum_{\epsilon= \pm 1} \sum_{\substack{p^{3} N\left|D_{1}\right| D_{2} \\
l_{1} n_{1} D_{2}=d_{2} m_{1} D_{1}^{2}}} \frac{\tilde{S}\left(\epsilon l_{2} n_{2}, l_{1} n_{1}, d_{2} m_{2}, D_{1}, D_{2}\right)}{D_{1} D_{2}} \tilde{\mathcal{J}}_{\epsilon ; F_{i}}\left(\sqrt{\frac{l_{1} n_{1} l_{2} n_{2} d_{2} m_{2}}{D_{1} D_{2}}}\right) \\
& \Sigma_{6}=\max _{i} \max _{M \leqslant N^{1 / 2+\varepsilon}} \frac{N^{2+\varepsilon}}{M^{3}} \sum_{\substack{d_{1}, d_{2}, d_{3} \\
\left(d_{1} d_{2} d_{3}, p\right)=1}} \sum_{\substack{n_{1}, n_{2} \sim M / d_{1} d_{2} \\
m_{1}, m_{2} \sim M / d_{1} d_{3} \\
l_{1}, l_{2} \sim M / d_{2} d_{3}}} \bar{\chi}\left(n_{1} l_{1} m_{2}\right) \chi\left(m_{1} n_{2} l_{2}\right) \\
& \sum_{\epsilon_{1}, \epsilon_{2}= \pm 1} \sum_{p^{3} N\left|D_{1}, p^{3} N\right| D_{2}} \frac{S^{\left(p^{3} N\right)}\left(\epsilon_{2} d_{2} m_{1}, \epsilon_{1} l_{2} n_{2}, l_{1} n_{1}, d_{2} m_{2}, D_{1}, D_{2}\right)}{D_{1} D_{2}} \mathcal{J}_{\epsilon ; F_{i}}\left(\frac{\sqrt{l_{2} n_{2} d_{2} m_{2} D_{1}}}{D_{2}}, \frac{\sqrt{l_{1} n_{1} d_{2} m_{1} D_{2}}}{D_{1}}\right) .
\end{aligned}
$$

It is easy to see that $|\Delta|+\left|\Sigma_{4}\right|+\left|\Sigma_{5}\right| \ll N^{2+\varepsilon}$ : indeed, by a divisor argument we have

$$
\Delta \ll \max _{M \leqslant N^{1 / 2+\varepsilon}} \frac{N^{2+\varepsilon}}{M^{3}} \sum_{d_{1}, d_{2}, d_{3}} \sum_{\begin{array}{c}
n_{1}, n_{2} \sim M / d_{1} d_{2} \\
m_{1}, m_{2} \sim M / d_{1} d_{3} \\
l_{1}, l_{2} \sim M / d_{2} d_{3}
\end{array}} \delta_{n_{1} l_{1}=n_{2} l_{2}} \ll \max _{m_{2}=m_{1}} \frac{N^{2+\varepsilon}}{M^{3}} \sum_{d_{1}, d_{2}, d_{3}} \frac{M^{3}}{\left(d_{1} d_{2} d_{3}\right)^{2}} \ll N^{2+\varepsilon}
$$

as desired. For $\Sigma_{5}$ we simply observe that the conditions $p^{3} N\left|D_{1}\right| D_{2}$ and $n_{1} l_{1} D_{2}=m_{1} d_{2} D_{1}^{2}$ implies $N \mid D_{1}$ and $N^{2} \mid D_{2}$ since $N \nmid n_{1} l_{1}$ is prime, so that $D_{1} D_{2} \geqslant N^{3}$, but $n_{2} l_{2} m_{2} d_{2} n_{1} l_{1} \ll M^{5} \ll N^{5 / 2+\varepsilon}$, so that (for sufficiently small $\varepsilon>0$ and sufficiently large $N$ ) we have $\Sigma_{5}=0$ by Lemma 3(a) (with $X_{1}=X_{2}=1$ ). Similarly one shows $\Sigma_{4}=0$.

The term corresponding to the long Weyl element can be bounded by

$$
\begin{aligned}
& \Sigma_{6} \ll \max _{M \leqslant N^{1 / 2+\varepsilon}} \frac{N^{1+\varepsilon}}{M^{3}} \sum_{\substack{d_{1}, d_{2}, d_{3} \\
\left(d_{2}, p\right)=1}} \sum_{\epsilon \in\{ \pm 1\}^{2}} \mid \sum_{\begin{array}{c}
n_{1}, n_{2} \sim M / d_{1} d_{2} \\
m_{1}, m_{2} \sim M / d_{1} d_{3} \\
l_{1}, l_{2} \sim M / d_{2} d_{3}
\end{array}} \bar{\chi}\left(n_{1} l_{1} m_{2}\right) \chi\left(m_{1} n_{2} l_{2}\right) \\
& \times \sum_{\substack{p^{3}\left|D_{1} \\
p^{3}\right| D_{2}}} \frac{S^{\left(p^{3}\right)\left(\epsilon_{2} \bar{N} m_{1} d_{2}, \epsilon_{1} \bar{N} n_{2} l_{2}, n_{1} l_{1}, m_{2} d_{2} ; D_{1}, D_{2}\right)}}{D_{1} D_{2}} \mathcal{J}_{\epsilon ; F}\left(\frac{\sqrt{n_{2} l_{2} m_{2} d_{2} D_{1}}}{N^{1 / 2} D_{2}}, \frac{\sqrt{n_{1} l_{1} m_{1} d_{2} D_{2}}}{N^{1 / 2} D_{1}}\right) \mid
\end{aligned}
$$


where $\mathcal{J}_{\epsilon ; F}$ satisfies the properties of Lemma 3(b). Here we used Lemma $6(\mathrm{~b})$ and (c) and note that the support of $\mathcal{J}$ given in Lemma $3(\mathrm{~b})$ implies

$$
D_{1}, D_{2} \ll \frac{M^{3} d_{2}}{N\left(d_{1} d_{2} d_{3}\right)^{2}} \ll N^{1 / 2+\varepsilon}
$$

so that automatically $\left(N, D_{1} D_{2}\right)=1$.

Remark: We pause for a moment and observe that the contribution of the terms $M=N^{1 / 2}, d_{1}=$ $d_{2}=d_{3}=1, D_{1}=D_{2} \asymp N^{1 / 2}$ without the character $\chi$ exhibits no essential cancellation and is of size $N^{5 / 2}$ as predicted by the contribution of the maximal Eisenstein series: indeed, the maximal Eisenstein series are parametrized by GL(2) cusp forms $f$ for $\Gamma_{0}(N)$, and a typical Fourier coefficient is given by $A(n, 1)=\sum_{d \mid n} \lambda_{f}(n)$; thus the maximal Eisenstein contribution is very roughly of the form

$$
\sum_{f}\left|\sum_{n \ll N^{1 / 2}} \frac{1}{\sqrt{n}} \sum_{d \mid n} \lambda_{f}(d)\right|^{6} \approx \sum_{f}\left|N^{1 / 4} L(1, f)\right|^{6} \approx N^{5 / 2} .
$$

In order to obtain the targeted bound $N^{2}$ we will have to use the extra oscillation of the character.

We apply Poisson summation in the 6 variables $n_{1}, n_{2}, m_{1}, m_{2}, l_{1}, l_{2}$. We call the dual variables $x_{1}, x_{2}, y_{1}, y_{2}, z_{1}, z_{2}$, respectively. By Lemma 3(b) the function

$$
J: n_{2} \mapsto \mathcal{J}_{\epsilon ; F}\left(\frac{\sqrt{n_{2} l_{2} m_{2} d_{2} D_{1}}}{N^{1 / 2} D_{2}}, \frac{\sqrt{n_{1} l_{1} m_{1} d_{2} D_{2}}}{N^{1 / 2} D_{1}}\right)
$$

satisfies

$$
n_{2}^{i} J^{(i)}\left(n_{2}\right) \ll_{i}\left(\frac{M^{3} d_{2}}{N\left(d_{1} d_{2} d_{3}\right)^{2} D_{2}}\right)^{i}
$$

for all $i \in \mathbb{N}_{0}$ under the present size conditions of the variables. We conclude that the dual variable $x_{2}$ can be bounded by

$$
\left|x_{2}\right| \leqslant N^{\varepsilon} \cdot \frac{D_{2}}{M / d_{1} d_{2}} \cdot \frac{M^{3} d_{2}}{N\left(d_{1} d_{2} d_{3}\right)^{2} D_{2}}=N^{\varepsilon} \frac{M^{2}}{N d_{1} d_{3}^{2}} \ll N^{\varepsilon}
$$

up to a negligible error. By a similar argument, the same bound holds for $x_{1}$, and we also have

$$
\left|y_{1}\right|,\left|y_{2}\right| \leqslant N^{\varepsilon} \frac{M^{2}}{N d_{1} d_{2} d_{3}} \ll N^{\varepsilon}, \quad\left|z_{1}\right|,\left|z_{2}\right| \leqslant N^{\varepsilon} \frac{M^{2}}{N d_{1}^{2} d_{3}} \ll N^{\varepsilon} .
$$

Now we can apply (3.5) with

$$
\alpha_{1}=\frac{x_{2} M}{d_{1} d_{2} D_{2}}, \quad \beta_{1}=\frac{y_{2} M}{d_{1} d_{3} D_{2}}, \quad \gamma_{1}=\frac{z_{2} M}{d_{2} d_{3} D_{2}}, \quad \alpha_{2}=\frac{x_{1} M}{d_{1} d_{2} D_{1}}, \quad \beta_{2}=\frac{y_{1} M}{d_{1} d_{3} D_{1}}, \quad \gamma_{2}=\frac{z_{1} M}{d_{2} d_{3} D_{1}}
$$

unless $x_{1} x_{2} y_{1} y_{2} z_{1} z_{2}=0$, in which case we apply (3.4) with $P=N^{\varepsilon}$ and

$$
A_{1}=\frac{\sqrt{M^{3} d_{2} D_{1}}}{d_{1} d_{2} d_{3} N^{1 / 2} D_{2}}, \quad A_{2}=\frac{\sqrt{M^{3} d_{2} D_{2}}}{d_{1} d_{2} d_{3} N^{1 / 2} D_{1}} .
$$


In this way we conclude by trivial estimates that

$$
\begin{aligned}
\Sigma_{6} \ll \max _{M \leqslant N^{1 / 2+\varepsilon}} & \frac{N^{1+\varepsilon}}{M^{3}} \sum_{\substack{d_{1}, d_{2}, d_{3} \\
\left(d_{2}, p\right)=1}} \sum_{\epsilon \in\{ \pm 1\}^{2}} \sum_{p^{3} \mid D_{1}, D_{2} \ll \frac{M^{3} d_{2}}{N\left(d_{1} d_{2} d_{3}\right)^{2}}} \frac{1}{D_{1} D_{2}} \\
& \times\left(\frac{\min \left(d_{1} d_{2}, d_{1} d_{3}, d_{2} d_{3}\right)\left(D_{1} D_{2}\right)^{1 / 2}}{M}+\frac{\left(d_{1} d_{2} d_{3}\right)^{2} N\left(D_{1}+D_{2}\right)}{M^{3} d_{2}}\right) \\
& \times \frac{M^{6}}{\left(d_{1} d_{2} d_{3}\right)^{4}} \sum_{\substack{\left|x_{1}\right|,\left|x_{2}\right| \ll N^{\varepsilon} \\
\left|y_{1}\right|,\left|y_{2}\right| \ll N^{\varepsilon} \\
\left|z_{1}\right|,\left|z_{2}\right| \ll N^{\varepsilon}}}\left|\widehat{S}_{\epsilon_{2} \bar{N}, \epsilon_{1} \bar{N}, d_{2}}^{\chi}\left(x_{1}, x_{2}, y_{1}, y_{2}, z_{1}, z_{2} ; D_{1}, D_{2}\right)\right|
\end{aligned}
$$

where $\widehat{S}$ was defined in (4.5). Notice that the $D_{1}, D_{2}$-sum restricts the $d_{2}$-variable to $d_{2} \ll N^{1 / 2+\varepsilon}$. By Corollary 10 we can bound the innermost sum by $N^{\varepsilon}\left(D_{1}, D_{2}\right)^{2} d_{2}^{2}\left(D_{1} D_{2}\right)^{-1}$, and obtain

$$
\Sigma_{6} \ll N^{2+\varepsilon} \sum_{\substack{d_{1}, d_{2}, d_{3} \\ d_{2} \ll N^{1 / 2+\varepsilon}}} \frac{1}{d_{1}^{2} d_{2} d_{3}^{2}} \sum_{D_{1}, D_{2} \ll N^{1 / 2+\varepsilon}} \frac{\left(D_{1}, D_{2}\right)^{2}\left(D_{1}+D_{2}\right)}{\left(D_{1} D_{2}\right)^{2}} \ll N^{2+\varepsilon}
$$

as desired. This completes the proof of Theorem 1.

\section{Proofs of Theorems $2-5$}

For the proof of Theorems 2, 3 and 5 we choose functions $F_{1}, \ldots, F_{J}$ as in Lemma 1 , and we apply Lemma 3 with $X_{1}=X_{2}=1$.

For the proof of Theorem 3 we proceed as follows. The outer sum is over cuspidal automorphic representations that we interpret as a sum over newvectors. We add artificially the oldforms and the Eisenstein spectrum and bound the mean value in question by

$$
N^{2+\varepsilon} \sum_{j=1}^{J} \int_{(N)}\left|\sum_{\substack{n \asymp C \\(n, N)=1}} A_{\varpi}(n, 1) \alpha(n)\right|^{2}\left|\left\langle F_{j}, \tilde{W}_{\mu_{\pi}}\right\rangle\right|^{2} \mathcal{N}(\varpi)^{-1} d \varpi .
$$

Here we used also (2.9). We open the square and apply the Kuznetsov formula. The diagonal term contributes $\ll N^{2+\varepsilon}\|\alpha\|^{2}$.

The contribution of the long Weyl element is bounded by

$$
N^{2+\varepsilon} \sum_{\substack{n, m \asymp X \\(n m, N)=1}}|\alpha(n) \alpha(m)| \sum_{\substack{N \mid D_{1}, D_{2} \\ D_{1}, D_{2} \ll X}} \frac{\left|S^{(N)}\left( \pm 1, \pm m, n, 1 ; D_{1}, D_{2}\right)\right|}{D_{1} D_{2}} \ll(N X)^{\varepsilon} X^{2} N^{1 / 2}\|\alpha\|^{2}
$$

by (4.2), since the support condition in Lemma $3(\mathrm{~b})$ with $X_{1}=X_{2}=1$ restricts $D_{1}, D_{2} \ll X$.

The contribution of the $w_{5}$ element is bounded by

$$
N^{2+\varepsilon} \sum_{\substack{n, m \asymp X \\(n m, N)=1}}|\alpha(n) \alpha(m)| \sum_{\substack{N\left|D_{1}\right| D_{2} \\ n D_{2}=D_{1}^{2} \\ D_{1} D_{2} \ll X^{2}}} \frac{\left|\tilde{S}\left( \pm m, n, 1 ; D_{1}, D_{2}\right)\right|}{D_{1} D_{2}},
$$

again by the support condition in Lemma 3(a). The summation condition implies $D_{1}=n d N, D_{2}=n d^{2} N^{2}$, which is only possible if $N \ll 1$, so that we obtain

$$
\sum_{n, m \asymp X}|\alpha(n) \alpha(m)| \sum_{d \ll 1} \frac{\left|\tilde{S}\left( \pm m, n, 1 ; n d N, n d^{2} N^{2}\right)\right|}{n^{2} d^{3}} \ll \sum_{n, m \asymp X}|\alpha(n) \alpha(m)| \ll X\|\alpha\|^{2}
$$


by Lemma 5 . This term is dominated by the $w_{6}$ contribution. A similar argument works for the $w_{4}$ contribution. This completes the proof.

A similar, but simpler, argument shows the bound in Theorem 2 .

The proof of Theorem 5 follows along the lines of [BBR, Theorem 2]. If $\left\|\alpha_{\pi}(p)\right\|_{\infty} \geqslant 1+\delta$, then $\lambda_{\pi}\left(p^{l}\right) \geqslant(l+1)(l+2)$ for some sufficiently large $l=l(\delta)$, see [BBR, (24)]. Hence for any $k \geqslant 1$ we have

$$
\#\left\{\pi \subseteq L_{\text {cusp }}^{2}\left(\Gamma_{0}(N) \backslash \mathbb{H}_{3}\right): \mu_{\pi} \in \Omega,\left\|\alpha_{\pi}(p)\right\| \geqslant 1+\delta\right\} \leqslant \sum_{\substack{\pi \subseteq L_{\text {cusp }}^{2}\left(\Gamma_{0}(N) \backslash \mathbb{H}_{3}\right) \\ \mu_{\pi} \in \Omega}} \frac{\left|A_{\varpi}\left(p^{l}, 1\right)\right|^{2 k}}{((l+1)(l+2))^{2 k}} .
$$

By $[\mathrm{BBR},(14)]$ we have

$$
\left|A_{\varpi}\left(p^{l}, 1\right)\right|^{2 k}=\sum_{r+s \leqslant 2 l k} \alpha_{r, s, l, k} A_{\varpi}\left(p^{r}, p^{s}\right) A_{\varpi}(1,1), \quad \sum_{r+s+\leqslant 2 l k}\left|\alpha_{r, s, l, k}\right| \leqslant\left(\frac{(l+1)(l+2)}{2}\right)^{2 k}
$$

so that by Cauchy-Schwarz and Theorem 2 the right hand side of (6.1) is bounded by

$$
\ll\left(N p^{2 l k}\right)^{\varepsilon} 2^{-2 k}\left(N^{2}+N^{1 / 2} p^{2 k l}\right)^{1 / 2} N \ll\left(N p^{2 l k}\right)^{\varepsilon} 2^{-2 k}\left(N^{2}+N^{5 / 4} p^{k l}\right) .
$$

Choosing $k=\left\lfloor\frac{3 \log N}{4 l \log p}\right\rfloor \geqslant 1$, we obtain

$$
\#\left\{\pi \subseteq L_{\text {cusp }}^{2}\left(\Gamma_{0}(N) \backslash \mathbb{H}_{3}\right): \mu_{\pi} \in \Omega,\left\|\alpha_{\pi}(p)\right\| \geqslant 1+\delta\right\} \ll N^{2+\varepsilon-\frac{3 \log 2}{2 l \log p}} .
$$

Finally we prove Theorem 4 . Here we choose sufficiently large parameters $X_{1}=X_{2}=X$ to be determined later and apply the Kuznetsov formula with the function $F^{(X, X)}$ as in (3.2). Then by Lemma 2 and (2.9) we have

$$
\sum_{\substack{\pi \subseteq L_{\text {cusp }}^{2}\left(\Gamma_{0}(N) \backslash \mathbb{H}_{3}\right) \\ \mu_{\pi}=(\rho+i \gamma, \rho-i \gamma,-2 i \gamma) \in \Omega \\|\rho| \geqslant \varepsilon}} X^{4+4\left|\Re \mu_{\pi}\right|} \ll N^{2+\varepsilon} \int_{(N)} \frac{\left|A_{\varpi}(1,1)\right|^{2}\left|\left\langle F^{(X, X)}, \tilde{W}_{\mu_{\pi}}\right\rangle\right|^{2}}{\mathcal{N}(\varpi)} d \varpi .
$$

The diagonal term contributes $N^{2+\varepsilon} X^{4}$. By Lemma 3(b), the long Weyl element contributes

$$
(N X)^{\varepsilon} N^{2} X^{4} \sum_{N \mid D_{1}, D_{2} \ll X^{2}} \frac{\left|S^{(N)}\left( \pm 1, \pm 1,1,1 ; D_{1}, D_{2}\right)\right|}{D_{1} D_{2}} .
$$

Assuming $X \leqslant N^{1-\varepsilon}$ and recalling that $N$ is prime, we have $N^{2} \nmid D_{1}, D_{2}$. Combining Lemma 6(b), Lemma 6(c) and Lemma 5 (with $N=1$ ), we obtain the bound

$$
(X N)^{\varepsilon} N^{2} X^{4} \sum_{D_{1}^{\prime}, D_{2}^{\prime} \ll X^{2} / N} \frac{N\left(D_{1}^{\prime} D_{2}^{\prime}\right)^{1 / 2+\varepsilon}\left(D_{1}^{\prime}, D_{2}^{\prime}\right)^{1 / 2}}{D_{1}^{\prime} D_{2}^{\prime} N^{2}} \ll(N X)^{\varepsilon} X^{6} .
$$

By Lemma $3(\mathrm{a})$ the $w_{5}$ element contributes

$$
(N X)^{\varepsilon} N^{2} X^{4} \sum_{\substack{N\left|D_{1}\right| D_{2} \\ D_{2}=D_{1}^{2} \\ D_{1} D_{2} \ll X^{3}}} \frac{\tilde{S}\left( \pm 1,1,1 ; D_{1}, D_{2}\right)}{D_{1} D_{2}}=0
$$

if $X \leqslant N^{1-\varepsilon}$. Similarly, the $w_{4}$ contribution vanishes. Choosing $X=N^{1-\varepsilon}$, we obtain the result. 


\section{ACKNOWLEDGEMENT}

The authors would like to thank the referee for a careful reading of the manuscript.

\section{REFERENCES}

[Ar] J. Arthur, Eisenstein series and the trace formula, in: Automorphic forms, representations and L-functions, Corvallis/Oregon 1977, Proc. Symp. Pure Math. 33 (1979), 253-274

[Ba] D. Balakci, Automorphic forms for congruence subgroups of $\mathrm{SL}(3, \mathbb{Z}), \mathrm{PhD}$ thesis, Göttingen 2015

[Bl] V. Blomer, Applications of the Kuznetsov formula on GL(3), Invent. math. 194 (2013), 673-729

[BBR] V. Blomer, J. Buttcane, N. Raulf, A Sato-Tate law for GL(3), Comm. Math. Helv. 89 (2014), 895-919

[BHM] V. Blomer, G. Harcos, P. Michel, Bounds for modular L-functions in the level aspect, Ann. Sci. Ecole Norm. Sup. 40 (2007), 697-740

[BKY] V. Blomer, R. Khan, M. Young, Mass distribution of holomorphic cusp forms, Duke Math. J. 162 (2013), 2609-2644

[Br] R. Bruggeman, Fourier coefficients of cusp forms, Invent. Math. 45 (1978), 1-18.

[BFG] D. Bump, S. Friedberg, D. Goldfeld, Poincaré series and Kloosterman sums for SL(3, Z), Acta Arith. 50 (1988), 31-89

$[\mathrm{Bu}] \quad$ J. Buttcane, Sums of $\mathrm{SL}(3, \mathbb{Z})$ Kloosterman sums, PhD thesis, UCLA 2012

$[\mathrm{DF}] \quad$ R. Dabrowski, B. Fisher, A stationary phase formula for exponential sums over $\mathbb{Z} / p^{m} \mathbb{Z}$ and applications to $G L(3)$ Kloosterman sums, Acta Arith. 80 (1997), 1-48

[DI1] J.-M. Deshouillers, H. Iwaniec, Kloosterman sums and Fourier coefficients of cusp forms, Invent. Math. 70 (1982/83), 219-288

[DFI] W. Duke, F. Friedlander, H. Iwaniec, The subconvexity problem for Artin L-functions, Invent. math. 149 (2002), 489-577

[DK] W. Duke, E. Kowalski, Large sieve inequalities for GL(n)-forms in the conductor aspect (with appendix by D. Ramakrishnan), Invent. math. 139 (2000), 1-39

[Fr] S. Friedberg, Poincaré series for GL $(n)$ : Fourier expansion, Kloosterman sums, and algebreo-geometric estimates, Math. Z. 196 (1987), 165-188

[Go] D. Goldfeld, Automorphic forms and L-functions for the group GL( $n, \mathbb{R})$, Cambridge studies in advanced mathematics 99 (2006)

[IK] H. Iwaniec, E. Kowalski, Analytic number theory, Colloq. Publ. 53, Amer. Math. Soc., Providence, RI, 2004.

[JPSS] H. Jacquet, I. Piatetski-Shapiro, J. Shalika, Conducteur des représentations du groupe linéaire, Math. Ann. 256 (1981), 199-214

[JS] H. Jacquet, J. Shalika, On Euler products and the classification of automorphic representations. I, Amer. J. Math. 103 (1981), 499-558

[Li] X. Li, Upper bounds on L-functions at the edge of the critical strip, IMRN 2010, 727-755

$[\mathrm{Ku}] \quad$ N. Kuznetsov, The Petersson conjecture for cusp forms of weight zero and the Linnik conjecture. Sums of Kloosterman sums, Math. USSR-Sb 39 (1981), 299-342.

[St] G. Stevens, Poincarè series on GL(r) and Kloostermann sums, Math. Ann. 277 (1987), 25-51

[Ve] A. Venkatesh, Large sieve inequalities for GL(n)-forms in the conductor aspect, Adv. in Math. 200 (2006), 336-356

Mathematisches Institut, Bunsenstr. 3-5, 37073 Göttingen, Germany

E-mail address: vblomer@math.uni-goettingen.de

Department of Mathematics, 244 Mathematics Building, University at Buffalo, Buffalo, NY 14260-2900 USA

E-mail address: jbuttcane@buffalo.edu

MTA Alfréd Rényi Institute of Mathematics, POB 127, Budapest H-1364, Hungary

E-mail address: maga.peter@renyi.mta.hu 